\title{
1 „Lay upon us the burden of the world's suffering". Quäkerhumanitarismus vor dem Ersten Weltkrieg
}

\section{Die Quäker in den USA. Eine Religionsgemeinschaft im Wandel}

1902 sprach Rufus Jones in Birmingham vor einer Versammlung von 300 britischen Quäkern über „Die Aufgabe unseres Zeitalters“ („The Task of our Age“). Jones, einer der bedeutendsten theologischen Erneuerer des Quäkertums in den USA und ein kommender Protagonist der Quäkerhilfe nach dem Ersten Weltkrieg, erläuterte seiner Zuhörerschaft, warum eine Religion nur dann Daseinsberechtigung besitze, wenn sie der Welt und ihren Problemen voll zugewandt sei. Angesichts der Not und des Elends könne sich niemand einen Anhänger Jesu Christi nennen, „if he stops at the salvation of his own soul“. Eine solche Haltung verwandele Religion „into a fine kind of selfishness“. Die Macht der Religion, so folgerte Jones, ,is measured not by what God has done for us, as by what God is doing through us"..1

In Jones' Worten spiegelte sich sowohl Umbruch als auch Kontinuität wider: Seine Zuhörerschaft verstand, dass seine Worte als Aufruf zur Öffnung gemeint waren, gerichtet an eine in ihrer Mehrheit immer noch stark nach innen gekehrte Glaubensgemeinschaft. Zur selben Zeit wollte Jones das kollektive Gedächtnis seiner Zuhörer aktivieren: an eine zu den Anfängen des Quäkertums zurückreichende „humanitäre“ Tradition, wie sie sich etwa in der Anti-Sklaverei-Bewegung und der Beteiligung an den verschiedensten Reformanliegen manifestiert hatte. Jones sprach über eine Glaubensgemeinschaft, die während ihrer zum Zeitpunkt seiner Rede rund 300 Jahre alten Geschichte viele Häutungen durchlaufen hatte. Die Religious Society of Friends war in ihren Ursprüngen eine der vielen puritanischen Sekten von britischen Dissenters gewesen, die sich im 17. Jahrhundert unter der Führung von George Fox (1624-1691) von der Church of England abspalteten. Während der Ära der Verfolgungen, die über weite Teile des 17. Jahrhunderts andauerte, floh eine größere Gruppe in die Neue Welt. Unter denen, welche die Heimat verließen, war auch der wohlhabende Kaufmann William Penn (1644-1718), der in der nach ihm benannten Kolonie Pennsylvania eine Art Quäker-Utopia gründete, mit dem Zentrum in Philadelphia, der „Stadt der brü-

1 Rufus Jones, The Task of our Age, 10/09/1902, zitiert nach John Ormerod Greenwood, Quaker Encounters Vol. 1: Friends and Relief (York: William Sessions Limited, 1975), 173.

Ә OpenAccess. ( 2022 Daniel Maul, publiziert von De Gruyter. [(c) BYY-NC-ND Dieses Werk ist lizenziert unter einer Creative Commons Namensnennung - 4.0 International Lizenz. 
derlichen Liebe“. Später gründeten sich Quäkergemeinschaften auch in anderen Teilen des britischen Empire, in Australien, Neuseeland und Südafrika, später auch in den afrikanischen und asiatischen Kolonien. Schließlich existierten kleine Quäkergemeinden auch außerhalb des angloamerikanischen Sprach- und Einflussraums, etwa in Deutschland, den skandinavischen Ländern oder in Böhmen. Noch bis in die zweite Hälfte des 20. Jahrhunderts freilich lebte die überwältigende Mehrzahl der Quäker in Großbritannien und den USA. ${ }^{2}$

Während der langen Zeit vom 17. Jahrhundert bis in die Gegenwart verstanden sich Quäker auf der ganzen Welt als Vertreter einer gemeinsamen Glaubensrichtung, die theologische wie weltanschauliche Überzeugungen und Haltungen miteinander verband. Zur selben Zeit gab es signifikante Unterschiede in den Entwicklungen des Quäkertums, die das Mutterland England und die Glaubensgenossen in den USA voneinander trennten. Während des 19. Jahrhunderts entwickelten sich auf beiden Seiten des Atlantiks relativ unabhängige Zweige liberaler, konservativer und evangelikaler Quäker, die jeweils unterschiedliche transnationale Netzwerke unterhielten. Während die britischen Quäker jedoch stets ihre formale Einheit beibehielten, repräsentiert durch die Existenz eines zentralen London Yearly Meeting, ${ }^{3}$ das allen Zweigen ein gemeinsames organisatorisches Dach bot, durchliefen die US-amerikanischen Quäker eine Reihe von Spaltungen. Diese sind in engem Verhältnis zu den großen religiösen Strömungen der Zeit zu sehen: zu den evangelikalen revivals und der aus ihr hervorgegangenen holiness-Bewegung sowie zum social gospel, der evangelikale innere Mission mit sozialer Aktion verband. Ein Teil wiederum schloss sich an einen aufkommenden modernistisch-liberalen Protestantismus an, der für eine kritisch-historische Theologie plädierte. Für die Quäker in den USA manifestierten sich diese Unterschiede anders als in Großbritannien auch in einer institutionellen Trennung. Eine Gruppe um Elias Hicks aus Long Island, die sogenannten Hicksites, wandten sich in den 1820er Jahren von der Mehrheitsgemeinde ab - im Wesentlichen als Reaktion auf den wachsenden Einfluss evangelikaler Strömungen innerhalb der

2 In der Gegenwart stellen afrikanische Gemeinden den mit Abstand größten Anteil an den weltweit etwa 300.000 praktizierenden Quäkern. Einen Überblick über die Entwicklung der Society of Friends auf beiden Seiten des Atlantiks und darüber hinaus bis in die Gegenwart liefert: John Punshon, Portrait in Grey: A Short History of the Quakers (London: Quaker Books, 1984). Siehe daneben auch Robynne Rogers Healey, „History of Quaker Faith and Practice 1650 - 1808“, in: Stephen W. Angell und Pink Dandelion (Hg.), The Cambridge Companion to Quakerism (Cambridge: Cambridge University Press, 2018), 13-31; Hamm und Barnes May, „Conflict and Transformation, 1808-1920“; Burdick und Dandelion, „Global Quakerism 1920 - 2015“.

3 Die Jahresversammlungen (Yearly Meetings) stellen die höchste Organisationsebene der Quäkerschaft dar. 
Gesellschaft. Ihre fundamentalistische Rückkehr zu den „Wurzeln“, ihre Betonung des inner light, bildeten langfristig einen wichtigen Impuls bei der Herausbildung eines theologisch liberalen Flügels. Auf der anderen Seite stand ab diesem Zeitpunkt die Mehrheit der Gemeinden, die unter dem Begriff der Orthodox Quakers zusammengefasst werden. Viele der orthodoxen Quäker gerieten unter den Einfluss evangelikaler Strömungen und näherten sich damit in vielfacher Weise dem protestantischen Mainstream in den USA an. Im Gegensatz zu der eher „basisdemokratischen“ Verfassung der Hicksites brachen einige orthodoxe Gemeinden auch mit der traditionellen Gottesdienstordnung der Quäker, die weder festangestellte Pastoren noch eine festgesetzte Liturgie kannte. Der Gegensatz zwischen „programmed“ und „unprogrammed“ Gottesdiensten, also solchen mit und ohne einen Pfarrer sowie einer festen Liturgie war dann auch das deutlichste Zeichen der Spaltung innerhalb der Gesellschaft bis ins 20. Jahrhundert. Daneben brachte die Annäherung der Orthodoxen an andere protestantische Denominationen auch institutionelle Neuerungen. Quäker eröffneten, so wie andere protestantische und katholische Gemeinden, Sonntagsschulen, errichteten eigene Colleges (wie Haverford und Swarthmore bei Philadelphia oder Earlham in Richmond, Indiana) und eröffneten zuletzt auch eigene Missionsgesellschaften. ${ }^{4}$

Eine weitere Spaltung ergab sich entlang geografischer Trennlinien. Eine zunehmende Kluft bestand hier zwischen dem Quäker-„Establishment“ an der Ostküste und den Gemeinden in den Staaten des Mittleren Westens wie Indiana, die politisch konservativer waren und sich gleichzeitig offener für evangelikale Einflüsse zeigten. ${ }^{5}$ Diese geografisch und theologisch getrennten Teile bewahrten sich einen kleinen Kern gemeinsamer Überzeugungen, ein „Quäker-Minimum“, das etwa das Priestertum aller Menschen betraf, den Glauben an das inner light beziehungsweise „Das von Gott in jedem Menschen“ und bis zu einem gewissen Grad auch die zentrale Stellung der testimonies (wie etwa das Friedenszeugnis, das Zeugnis der Einfachheit, der Integrität oder der Gleichheit), durch die die Quäker die Präsenz Gottes in der Welt bezeugen. Dagegen unterlagen bestimmte überlieferte äußere Attribute wie eine quäkerspezifische schlichte Kleidung, Enthaltsamkeit bei Alkohol, Tabak und anderen Rauschmitteln oder das Verbot von Tanz oder Gesang einem permanenten Aushandlungsprozess. Diese Fluidität erfasste im 19. Jahrhundert schließlich auch Kernbereiche quäkerlicher Theologie und Praxis wie etwa die präzise Auslegung des „Friedenszeugnisses“, das heißt der Verpflichtung zu absoluter Gewaltfreiheit und einem radikalen Pazifismus,

4 Hamm und Barnes May, „Conflict and Transformation, 1808-1920“; weiterführend zur Spaltung H. Larry Ingle, Quakers in Conflict : The Hicksite Reformation, (Knoxville: University of Tennessee Press, 1986).

5 H. Larry Ingle, Quakers in Conflict: The Hicksite Reformation. 
oder die Verweigerung von bestimmten Loyalitätsbezeugungen gegenüber staatlichen Autoritäten - etwa das Verbot, Eide zu leisten. In beiden Fällen wuchs das Spektrum der Auslegungen und die Spannbreite der Interpretationen, und die Starrheit wich einer gewissen Flexibilität, wobei es gerade diese Offenheit war, die Quäker der unterschiedlichen Richtungen in teils erbitterte Streitigkeiten führte. ${ }^{6}$

Eine der am heftigsten umstrittenen Fragen war dabei, inwieweit sich Quäker dem allgemeinen protestantischen Trend zur Gründung von Missionsgesellschaften zur Verbreitung des Glaubens anschließen sollten beziehungsweise durften. Hier gingen die Vorstellungen weit auseinander. Während viele Quäker Ende des 19. Jahrhunderts in Großbritannien und den USA dem evangelikalen Impuls folgten und eigene Missionsgesellschaften gründeten und selbst zu Missionaren wurden, wurde die Missionsbewegung selbst auf beiden Seiten des Atlantiks niemals ein vollständig akzeptierter Teil der Society of Friends. Wenngleich evangelikale Quäkermissionare bei einigen der humanitären Unternehmungen eine Schlüsselrolle spielen sollten, blieb die Haltung des offiziellen Quäkertums zögerlich und oftmals sogar feindselig. Wie in diesem Buch zu zeigen sein wird, entwickelten sich die humanitären Unternehmungen der Quäker im 20. Jahrhundert - aus unterschiedlichen Gründen - in erster Linie in Abgrenzung zum Evangelikalismus und der Missionsbewegung. Dies bedeutete freilich nicht, dass sich die Quäkerhilfe frei von missionarischen Elementen entwickelte. Im später insbesondere in Abgrenzung zum relief work beziehungsweise service work verwendeten Begriff der message work sollte diese Ambiguität zum Tragen kommen.

Evangelikalismus und Missionsbewegung standen in einer weiteren Hinsicht im Hintergrund Pate bei der Entwicklung eines quäkerspezifischen Humanitarismus. Das Aufkommen einer Bewegung junger Friends, des Young Friends Movement (YFM) in den späten 1890er Jahren war in vielfacher Hinsicht die Antwort auf die tiefgreifende Herausforderung, vor welche die evangelikale Bewegung die Quäker insgesamt stellte. Im Gegensatz zur Dynamik und Mobilisationskraft des Evangelikalismus erschienen vielen jungen Quäkern - über die Trennung in $\mathrm{Hi}$ cksites und Orthodoxe hinweg - ihre eigenen Gemeinden als konservativ und in Traditionen erstarrt. Anfänglich selbst von einem evangelikalen Impuls angetrieben, war es ihr Ziel, dem Quäkertum neues Leben einzuhauchen und es damit vor dem Verschwinden in der Bedeutungslosigkeit zu bewahren. ${ }^{7}$ Das YFM spiegelte als Bewegung junger Quäker innerhalb der Society of Friends in einem weiteren Sinn den Zeitgeist wider: Die Hauptinspiration kam von anderen pro-

6 Siehe hierzu vertieft Thomas D. Hamm, The Transformation of American Quakerism : Orthodox Friends, 1800-1907 (Bloomington: Indiana University Press, 1988).

7 Greenwood, Friends and Relief, $166 \mathrm{f}$. 
testantischen Vereinigungen wie der Young Men's Christian Association (YMCA), dem Christian Endeavour oder der Student Volunteer Union (SVU), die allesamt gezielt junge Männer und Frauen für die Erneuerung der offiziellen protestantischen Religion aus dem Geist des Evangelikalismus gewinnen wollten und sich missionarischen Aktivitäten widmeten. Gemeinsam war diesen Vereinigungen, dass sie einen neuartigen Raum innerhalb und quer $\mathrm{zu}$ den etablierten Religionsgemeinschaften schufen, und direkt auf Jugendliche und junge Erwachsene zielten. Besonders attraktiv war, dass es sich um Bewegungen handelte, die relativ autonom von der Hierarchie beziehungsweise der Geriarchie der Kirche aus der Mitte der Jugend selbst kam. Diesen Impetus teilten sie etwa mit der zur selben Zeit entstehenden säkularen Deutschen Jugendbewegung, die ihrerseits einen Bezugspunkt für viele Quäker vor allem in England darstellte. ${ }^{8}$ Der Einfluss der YMCA und der SVU, die mit missionarischem Eifer an die „Evangelisierung der Welt in dieser Generation“ gingen, auf junge Quäker kann kaum überschätzt werden. Einige der zukünftigen prominenten Mitglieder des AFSC, insbesondere solche aus dem Mittleren Westen, waren von diesem Einfluss geprägt. Clarence Pickett, der langjährige Executive Secretary des Komitees, war einer von ihnen gewesen und hatte zu Anfang eine missionarische Laufbahn erwogen. Ideen der „muscular christianity“ und einer „lebendigen Religion“ und ein Gefühl von Abenteuerlust, die mit der Missionsbewegung verbunden waren, übten zweifellos einen starken Reiz auf alle jene aus, die den engen Grenzen des Gemeindelebens in der Provinz entkommen wollten. ${ }^{9}$

An diesem Punkt setzte das YFM an, indem es versuchte, den Erneuerungswillen für junge Quäker in eine andere Richtung zu lenken. In England lag die Initiative bei einer Gruppe um John Wilhelm Rowntree (1868-1905), dem Erben der Birminghamer Rowntree-Schokoladendynastie. In den USA waren es vorwiegend jüngere Dozenten an den Quäker-Colleges Haverford, Swarthmore und Earlham, die die Bewegung leiteten. Zentral unter Letzteren war insbesondere der bereits erwähnte Rufus Jones (1863-1948), der „new prophet“ der Quäker, der 1894 Herausgeber der Monatsschrift The American Quaker wurde, die er nutzte,

8 Hollinger, Protestants Abroad: How Missionaries Tried to Change the World but Changed America; Porterfield, „Protestant Missionaries: Pioneers of American Philanthropy“. Zum World Christian Student Movement Charles Howard Hopkins, John R Mott 1865-1955: A Biography (Grand Rapids: William B. Eerdmans, 1980).

9 Lawrence McK. Miller, Witness for Humanity: A Biography of Clarence E. Pickett (Wallingford, PA: Pendle Hill Publications, 1999); Hamm und Barnes May, „Conflict and Transformation, 18081920“; Greenwood, Quaker Encounters Vol. 2: Vines on the Mountains, 204-252; Greenwood, Quaker Encounters Vol. 3: Whispers of Truth, 1-188. 
um seine Ideen der Erneuerung und Einheit des Quäkertums zu verbreiten. ${ }^{10}$ Die Ideen von Rowntree und Jones (die in engem und freundschaftlichem Kontakt miteinander standen) führten zu einer Wiederbelebung des Quäkertums aus dem Geist einer mystisch geprägten, auf die persönliche religiöse Erfahrung konzentrierten modernistischen Theologie. Daneben spielte vor allem soziales Bewusstsein und Aktion eine zentrale Rolle im Denken dieser Reformer. Sie interpretierten die Idee des ,inneren Lichts“ im Sinne einer religion of deeds not words. Gegen den evangelikalen Aufruf der Seelenrettung setzten sie den Begriff des service, des allgemeinen und umfassenden Dienstes an der Menschheit im Inneren und Äußeren. Die neue Gemeinschaft war eine fellowship of service. ${ }^{11}$ Es ging um sozialen Dienst im praktischen Sinn, jedoch aufgeladen mit einer nicht weniger religiösen Bedeutung als der missionarische Eifer der Evangelikalen. Für die Entwicklung eines quäkerspezifischen humanitären Dienstes war dieses Postulat wegweisend. Rufus Jones fasste diese Ideen 1902 vor einer britischen Zuhörerschaft zusammen: „We have to make this terrestrial paradise before we get any celestial city. The great task we are about to devote ourselves to is to be not so much personal salvation as the broad mission of social righteousness (...) the task of carrying holiness into the very structure of human society. “ Mit dieser Interpretation standen die Quäker für eine weltliche Religion und der beste Weg, Gott zu ehren, bestand darin, das Leiden der Welt zu lindern. John Wilhelm Rowntree machte deutlich, dass dieses Verständnis von Service keine nationalen Grenzen kannte. Sein abschließendes Gebet auf einer Konferenz in Manchester im Jahr 1895 war in diesem Sinne programmatisch: „Lay upon us the burden of the world's suffering. “12

Von Anbeginn war das YFM eine transnationale Bewegung. Quäker wie Jones und Rowntree kannten sich gut und pflegten regen Austausch miteinander. Sie nutzten dabei alteingesessene Netzwerke und knüpften neue. Sie trafen sich in gemeinsamen Sommerlagern, etwa in der neu eingerichteten Quäkerbildungsstätte Woodbroke bei Birmingham. Der Einfluss des YFM machte sich in den Jahren vor dem Ersten Weltkrieg zuerst auf nationaler Ebene bemerkbar und brachte Quäker aller Richtungen mit einer Vielzahl von sozialen Reformunter-

10 Stephen Allott, John Wilhelm Rowntree (1868-1905) (York: Sessions of York, 1994); Zu Rufus Jones allgemein ist einschlägig (und mit einer reichen Bibliographie ausgestattet): Claus Bernet, Rufus Jones (1863-1948) (Frankfurt am Main: Peter Lang, 2009); daneben die klassische Biografie von David Hinshaw, Rufus Jones, Master Quaker (New York: Putnam1951); Guy Aiken, „Beyond Liberalism: Rufus Jones and Thomas Kelly in the History of Liberal Religion, “Quaker Theology 11, 2 (2012).

11 Greenwood, Friends and Relief, 172.

12 Greenwood, Friends and Relief, 177. 
nehmungen in Verbindung. Wenngleich viele Quäkergemeinden ihren konservativen Charakter beibehielten, trug das YFM dazu bei, dass die Quäker in der öffentlichen Wahrnehmung zunehmend als Teil des sozialen Protestantismus galten.

Wieder gab es signifikante Unterschiede zwischen den USA und Großbritannien. Während die „modernistische“ Bewegung in Großbritannien einen Marsch durch die Institutionen antrat, der vor dem Weltkrieg schon weit fortgeschritten war, war der Weg in den USA angesichts der vielfältigen Zersplitterung steiniger. Dies schmälerte freilich nicht den Einfluss des YFM. Gerade vor dem Hintergrund der tiefen Risse, die die verschiedenen Gruppen von Quäkern voneinander trennten, leistete das YFM seinen wichtigsten Beitrag. Als eine der wenigen, wenn nicht als einzige Institution innerhalb der Quäkerschaft in den USA, schuf es ein Gefühl der Verbundenheit und bot Gelegenheit zu Austausch und Zusammenarbeit junger Quäker über alle Trennlinien hinweg. Damit bereitete das YFM den Boden für die Zusammenarbeit in humanitären Hilfsaktionen während des Ersten Weltkriegs. ${ }^{13}$

Zuletzt stieß das YFM eine neue Diskussion um die Bedeutung des peace testimony für die Quäker an. Das Spektrum der Auslegungen war breit und erstreckte sich von einer radikalen und absolut gesetzten bis hin zu einer pragmatischen und eher abstrakten Interpretation. Entsprechend reichten die praktischen Schlussfolgerungen, die aus dem peace testimony gezogen wurden, von Positionen, die Gewalt unter allen Umständen ablehnten, bis hin zu solchen, die Gewalt als eine gegebene Tatsache des internationalen Lebens akzeptierten und Frieden eher als ideales Ziel denn als unmittelbares Gebot betrachten. Über weite Teile des 19. Jahrhunderts neigten viele Freunde letzterer Position zu. In den Jahren vor dem Ersten Weltkrieg hatte das YFM entscheidenden Anteil daran, dass eine radikale und unbedingte Auslegung des Friedenszeugnisses an Stärke gewann. „Let us have done with an easy religion“, rief einer der australischen Delegierten einer All-Quaker-Konferenz im englischen Swanwick, 1911 einer internationalen Zuhörerschaft junger Quäker zu und verband dies mit dem Aufruf zur „Rückkehr“ zu einer absoluten Auslegung des Friedenszeugnisses. ${ }^{14}$

Obwohl sie weit davon entfernt waren, eine Mehrheit der Quäker hinter sich zu versammeln, wurden Quäker auf beiden Seiten des Atlantiks nach Beginn des Krieges zur treibenden Kraft hinter der Gründung von Friedensgesellschaften wie

13 Hamm und Barnes May, „Conflict and Transformation, 1808-1920“.

14 Greenwood, Friends and Relief, 177. 
der Fellowship of Reconciliation, die sich für kompromisslosen Pazifismus selbst in Zeiten nationaler Kriegshysterie einsetzten. ${ }^{15}$

\section{Die humanitären Quäker}

Ein Merkmal, das Quäker nicht nur in den USA, sondern auch über die nationalen Trennlinien hinweg mit den britischen Glaubensgenossen zu verbinden scheint, ist die gemeinsame humanitäre Tradition. Weitgehend anerkannt ist die Schlüsselposition, die einzelne Friends in mehreren Ländern in der Anti-Sklaverei-Bewegung einnahmen. Darüber hinaus waren Quäker von beiden Seiten des Atlantiks und oft im Austausch miteinander an den verschiedensten philanthropischen und humanitären Unternehmungen beteiligt - von der reformpädagogischen Umgestaltung des Erziehungswesens über die Reform des Gefängniswesens ${ }^{16}$ bis zur Temperenzbewegung zum Kampf gegen Prostitution und „White Slavery“, und besonders prominent in allen Zweigen der nationalen und internationalen Friedensbewegung ${ }^{17}$. Viele Quäkerinnen standen an der Spitze der internationalen Bewegung für die Rechte der Frauen. ${ }^{18}$ In den USA setzten sich die Quäker nicht nur für befreite Sklaven ein, sondern kümmerten sich auch in der Tradition von William Penn um die indigenen Völker des Kontinents. Während der Industrialisierung waren Quäker auch unter den Pionieren einer „social christianity“ $z$ u finden. ${ }^{19}$

Dessen ungeachtet: Der Fokus auf die teils lange zurückreichende gemeinsame Tradition von britischen und amerikanischen Quäkern, insbesondere in der Anti-Sklaverei-Bewegung, verstellt zuweilen den Blick dafür, dass eine durchgehende humanitäre Linie, welche die Geschichte der Religious Society of Friends vom 18. ins 20. Jahrhundert verbindet, zumindest was die USA betrifft, ein Stück invention of tradition darstellt. Unter den Urhebern dieser erfundenen Tradition ungebrochenen humanitären Engagements spielte das American Friends Service

15 Zum britischen Fall: Thomas C. Kennedy, „Fighting About Peace: The No-Conscription Fellowship and the British Friends Service Committee, 1915-1919“, in: Quaker History 33, 2, (1980) $3-22$.

16 Elizabeth Gurney Fry und Gil Skidmore, Elizabeth Fry: A Quaker Life. Selected Letters and Writings, The Sacred Literature Series (Lanham, MD: AltaMira Press, 2005).

17 So zum Beispiel sichtbar bei David Cortright, Peace: A History of Movements and Ideas (Cambridge: Cambridge University Press, 2008); Charles Chatfield, The American Peace Movement: Ideals and Activism (New York: Twayne Publishers, 1992).

18 Margaret Hope Bacon, Mothers of Feminism: The Story of Women in America (Philadelphia: Harper and Row, 1986); Sybil Oldfield, Doers of the Word: British Women Humanitarians 19001950 (London, 2006).

19 Hamm und Barnes May, „Conflict and Transformation, 1808-1920“. 
Committee selbst, wie zu zeigen sein wird, eine nicht unerhebliche Rolle. Für das 19. Jahrhundert trifft die Behauptung dieser Tradition jedoch allenfalls auf Individuen und kleinere Gruppen innerhalb der Quäkerschaft zu. Während die Society of Friends in England bei mehreren Gelegenheiten während des 19. Jahrhunderts Hilfsaktionen organisierte - vom griechischen Unabhängigkeitskrieg über die irische Hungersnot bis zum preußisch-französischen Krieg 1870/71 - war dies in den USA nicht der Fall. ${ }^{20}$ Der ursprüngliche Impuls hinter der Hicksites-Abspaltung war im Gegenteil sogar die Unzufriedenheit mit der „Verweltlichung“ der Gemeinden, die sich aus Sicht dieser Gruppe gerade in der Zusammenarbeit mit den verschiedensten Reformanliegen und den Abolitionisten außerhalb der Society of Friends manifestierte. Die „Rückkehr“ zu den Kerninhalten des Quäkerglaubens und die Betonung innerweltlicher religiöser Praxis geschah zunächst in Abgrenzung zu einer als Aufweichung und Verlust erlebten Realität. Aber auch unter orthodoxen Quäkern waren solche Öffnungen zur Welt, die sich in Bündnissen mit den unterschiedlichsten Reformgruppen offenbarten, nicht unumstritten und daher wurden sie zunächst auch längst nicht von allen nachvollzogen. $^{21}$

Vielmehr ist richtig, dass die unterschiedlichen Richtungen der Quäker im Verlauf des 19. Jahrhunderts auf ihre jeweils eigene Weise zu einem stärkeren Engagement mit humanitären und reformerischen Anliegen gelangten, was dann in vielen Fällen zu einer Zusammenarbeit mit anderen gleichgesinnten Gruppen außerhalb der eigenen Gemeinschaft führte. So lagen bei den Hicksites mit ihrer radikaleren Auslegung der Quaker testimonies die Wurzeln einer gegen Mitte des Jahrhunderts hervortretenden Gruppe von „progressive friends“, die durch „utmost liberty to individual conscience“ und gerade dadurch zur Unterstützung von „every imaginable reform, from nonresistance to abolition to temperance to women's rights“ gelangten. Einen ersten institutionellen Ausdruck fand der humanitäre Impetus der Hicksites in der Gründung der Friends Union for Philanthropic Labor 1882, der gegen Ende des 19. Jahrhunderts alle Hicksites-Gemeinden angehörten.22 Ihre Bündnispartner reichten bis in die Kreise utopischer Sozialisten wie Robert Owen. Auch radikale Sklavereigegnerinnen wie Lucrezia Mott kamen aus dieser Richtung.

Mit Blick auf die Rolle von Frauen wie Mott knüpften Quäkerinnen (aller Richtungen) im Übrigen an eine lange Tradition an. Anders als in den meisten

20 Greenwood, Friends and Relief, 11-164; zu Irland, siehe auch ausführlich Norbert Götz, Georgina Brewis, and Steffen Werther, Humanitarianism in the Modern World: The Moral Economy of Famine Relief, (New York: Cambridge University Press, 2020).

21 Hamm und Barnes May, „Conflict and Transformation, 1808-1920“.

22 Hamm und Barnes May, „Conflict and Transformation, 1808-1920“, 34. 
protestantischen Gemeinschaften (vom Katholizismus ganz zu schweigen) spielten Frauen innerhalb der Quäkergemeinden früh eine aktive und in wesentlichen Fragen religiöser Praxis auch gleichgestellte Rolle. Das women's ministry war weit verbreitet und etabliert, was Frauen in der Society of Friends in einem Maß Zugang zur öffentlichen Sphäre eröffnete, das sie von anderen Frauen unterschied und zu einem Teil die überproportionale Vertretung von Quäkerinnen in verschiedenen Reformbewegungen gegen Sklaverei, für Frieden und die Rechte der Frauen erklärt. ${ }^{23}$

Zur selben Zeit fanden auch orthodoxe Quäker über andere Wege zu humanitären Unternehmungen aller Art. Als besonders bedeutend erwies sich dabei die Zusammenarbeit mit evangelikalen Gruppen, die sie zum Teil eines eng gesponnenen Netzes der Missionsarbeit, etwa in China oder im Mittleren Osten werden ließen. Wenngleich amerikanische Quäker somit über ein „Tor zur Welt“ verfügten, das ihren Blick über die Grenzen der USA hinaus weitete, ${ }^{24}$ waren ihnen ihre britischen Glaubensgenossen zunächst weit voraus, wenn es um die ersten humanitären Einsätze über nationale Grenzen hinaus ging. Bereits während der Napoleonischen Kriege entsandten die Quäker in London Hilfe aufs Festland. Während des 19. Jahrhunderts waren britische Quäker unter den ersten, die Hilfskomitees gründeten, etwa zugunsten der Opfer des griechischen Unabhängigkeitskrieges gegen das Osmanische Reich während der 1820er Jahre, während der großen irischen Hungersnot in den 1840er Jahren oder anlässlich des Französisch-Deutschen Krieges 1870. Wenn amerikanische Quäker überhaupt an diesen frühen Bemühungen teilnahmen, so taten sie dies wie bei der Hilfe für Griechenland oder Irland als Einzelpersonen oder als Teil von größeren Hilfsaktionen. ${ }^{25}$ Erst in den 1890er Jahren begannen einige Quäkergemeinden an der Ostküste ein klareres humanitäres Profil zu entwickeln. Quäkerhilfskomitees bildeten sich zum Beispiel in Philadelphia anlässlich der großen Hungersnot im Russischen Reich 1891/1892. ${ }^{26}$ Beim Einsatz zugunsten der christlichen armenischen Minderheit im Osmanischen Reich, die 1894/1895 Opfer eines der wieder-

23 Brown und Stuard, Witnesses for Change: Quaker Women over Three Centuries. Hope Bacon, Mothers of Feminism: The Story of Women in America; Abigail Green, „Humanitarianism in Nineteenth Century Context: Religious, Gendered, National“, in: Historical Journal 57, 4 (2014), 11571175.

24 Tyrell, Reforming the World: The Creation of America's Moral Empire.

25 Curti, American Philanthropy Abroad, 22-64.

26 Curti, American Philanthropy Abroad , 99-132. Die Verbundenheit mit Russland rührte dabei auch von Verbindungen mit einigen russischen Sekten wie den Dukhobors her: Charlotte Alston, „,A Great Host of Sympathisers': The Doukhobor Emigration and Its International Supporters, 1895-1905“, in: Journal of Modern European History 12, 2 (2014), 200 -215. 
kehrenden Massaker geworden war, kam erstmals die missionarische Verbindung evangelikaler Quäker bei der Mobilisierung der Gesellschaft in den USA zum Tragen. ${ }^{27}$

Trotzdem machte die Hilfe für Notleidende und Verfolgte außerhalb der USA lange Zeit nur einen sehr kleinen Anteil der wohltätigen Aktivitäten der amerikanischen Quäker aus, die sich vor allem auf das eigene Land konzentrierten. Damit spielten die Quäker auch keine wichtige Rolle in der ersten Phase der humanitären Expansion der USA, die als integraler Bestandteil des amerikanischen Aufstiegs zur Weltmacht an der Wende vom 19. zum 20. Jahrhundert gilt. Im selben Maß, in dem die US-amerikanische Öffentlichkeit seit spätestens den 1890er Jahren in Emily Rosenbergs Worten nie mehr „without a foreign crisis to relieve“ war, ${ }^{28}$ wuchs auch das Interesse der Außenpolitik an den Aktivitäten privater Hilfsorganisationen im Ausland.

Insbesondere das 1881 gegründete Amerikanische Rote Kreuz nahm dabei eine Pionierfunktion ein. Als erste nationale Rotkreuzgesellschaft ging das ARC über das ursprüngliche Mandat des Roten Kreuzes hinaus, das sich gemäß der ersten Genfer Konvention von 1864 auf die Versorgung im Krieg verwundeter Soldaten beschränkte. Bereits in den 1890er Jahren wurde das ARC jenseits der amerikanischen Grenzen, in Russland und im Osmanischen Reich (zugunsten der Armenier), aktiv.

Nach dem Spanisch-Amerikanischen Krieg von 1898, der den Aufstieg der USA zur Kolonialmacht auf den Philippinen sowie zur uneingeschränkten Vormacht in der Karibik markierte, entdeckte die US-Regierung zunehmend den Wert privater humanitärer Hilfe. Sie wurde zu einem Mittel, diplomatische und handelspolitische Ziele abseits der offiziellen Außenpolitik zu verfolgen. Nach 1898 wurde es zur Regel, dass der Präsident (der ab 1905 automatisch Ehrenvorsitzender des ARC war) in einer Krise an die Öffentlichkeit appellierte, an das Rote Kreuz zu spenden.

Unter der Präsidentschaft Theodore Roosevelts wurden die Verbindungen noch enger: Während des mexikanischen Bürgerkriegs (1910 - 1920) etwa richtete das ARC ein nationales Hilfskomitee ein, in dem sowohl Vertreter der Regierung als auch Vertreter der Industrie mit wirtschaftlichen Interessen in Mexiko saßen. Umgekehrt nahm die US-Administration eine aktivere Rolle bei der finanziellen

27 Zur Quäkerpräsenz im Nahen Osten seit den 1870er Jahren siehe die Doktorarbeit von Catherine Baylin, „Quaker Activity in Ramallah: 1869-1914“ (The American University in Cairo, 2010). Außerdem Tusan, „The Business of Relief Work: A Victorian Quaker in Constantinople and Her Circle“.

28 Rosenberg, Spreading the American Dream: American Cultural and Economic Expansion, 1890-1945, 34. 
und logistischen Unterstützung privater Hilfsaktionen ein, etwa zugunsten der Opfer des großen Erdbebens in Messina 1908. Im Wesentlichen blieb die Kooperation aber inoffiziell - Hilfskampagnen und Hilfseinsätze waren weiterhin überwiegend privat organisiert und finanziert. Zur selben Zeit wurde deutlich, dass die amerikanische Außenpolitik das Potenzial der humanitären Hilfe als soft face amerikanischer Interessen nicht nur zu entdecken, sondern auch zunehmend zu nutzen wusste. Bei Ausbruch des Ersten Weltkriegs waren somit Mechanismen etabliert, die sich nach dem Kriegseintritt der USA zur Mobilmachung auf gesellschaftlicher Ebene nutzen ließen. ${ }^{29}$

Während das ARC, neben einigen anderen, kleineren religiösen oder ethnisch definierten (etwa italoamerikanischen) Gruppen, zum Teil eines inoffiziellen, öffentlich-privaten Hilfsnetzwerks wurde, standen die Quäker im Wesentlichen abseits. ${ }^{30}$ Die Gründe waren vielfältig: Eine traditionelle Skepsis gegenüber der Zusammenarbeit mit staatlichen Stellen kam ohne Zweifel ins Spiel. Während des Spanisch-Amerikanischen Krieges von 1898, der eine breite Diskussion um die Außenpolitik und insbesondere um den Status der USA als imperiale und koloniale Macht auslöste, stand ein Großteil der Quäker an der Ostküste im Lager der amerikanischen Anti-Interventionisten. Sie kritisierten überwiegend den Ansatz des pro-interventionistischen beziehungsweise imperialistischen Lagers, das expansive Vorstellungen von der außenpolitischen Rolle der USA mit der Forderung nach humanitären Interventionen kombinierte.

Zum Tragen kam in diesem Zusammenhang auch die Stärkung des peace testimony im Sinne eines absolut gesetzten, kompromisslosen Pazifismus, in dem sich der wachsende Einfluss des Young Friends Movement ausdrückte. Quäker waren ohnedies traditionell und teils prominent in allen Teilen der amerikanischen und internationalen Friedensbewegung vertreten. Dieser Einfluss wuchs nun weiter. Bereits vor dem Krieg etwa diente das Quäker-College Swarthmore als Zentrum des world peace through world law movement. ${ }^{31} 1914$ waren wiederum Quäker auf beiden Seiten des Atlantiks, darunter viele Young Friends, federfüh-

29 Irwin, Making the World Safe. The American Red Cross and a Nation's Humanitarian Awakening, 13-35.

30 Elisabeth Marie Piller, „To Aid the Fatherland: German-Americans, Transatlantic Relief Work and American Neutrality, 1914-17“, in: Immigrants \& Minorities 35, 3 (2017), 196-215. Für religiose Mobilisierung z.B. Granick, International Jewish Humanitarianism in the Age of the Great War. 31 J. William Frost, „Our Deeds Carry Our Message: The Early History of the American Friends Service Committee,“ Quaker History 81, no. 1 (1992), 5. 
rend bei der Gründung der Fellowship of Reconciliation, die für einen kompromisslosen Pazifismus auch in Kriegszeiten stand. ${ }^{32}$

32 Siehe für den britischen Fall Kennedy, „Fighting About Peace: The No-Conscription Fellowship and the British Friends Service Committee, 1915-1919“. 


\title{
2 Constructive Service. Der Erste Weltkrieg und die Gründung des AFSC
}

\begin{abstract}
We are united in expressing our love for our country and our desire to serve her loyally. We offer our services to the Government of the United States in any constructive work in which we can conscientiously serve humanity. ${ }^{1}$
\end{abstract}

Ende November 1918, rund zwei Wochen nach dem Ende des Ersten Weltkriegs, kamen die führenden Mitglieder des American Friends Service Committee in Philadelphia zusammen, um Bilanz zu ziehen. Der Ton des Treffens war enthusiastisch: Das AFSC und die humanitäre Arbeit, die junge Quäkerinnen und Quäker in den eineinhalb vorangegangenen Jahren an den europäischen Kriegsschauplätzen geleistet hatten, war eine Erfolgsgeschichte, darin waren sich alle einig. Insbesondere ein Aspekt stach hervor: Vor der Existenz des AFSC, so vermerkte das Protokoll, sei es jungen Quäkern nur durch den Dienst an der Waffe möglich gewesen, dem Vaterland „and its ideals“ gegenüber Loyalität zu zeigen und gleichzeitig im Gefühl zu handeln, ,work of national importance“ zu leisten. Nun jedoch sei durch die Arbeit des AFSC eine neue Zeit angebrochen - eine Zeit, in der ,without suspicions our young people can serve their country by taking the message of good-will to the war stricken zones“.2

Von solcher Zuversicht war noch wenig zu spüren gewesen, als eine kleine Gruppe von Quäkern im April 1917 in Philadelphia zusammengekommen war, um jene Institution zu gründen, die schließlich zum Gesicht der amerikanischen Quäkerhilfe werden sollte. ${ }^{3}$ Dem AFSC ging es anfänglich in erster Linie darum, Quäkern im wehrfähigen Alter die Möglichkeit zu eröffnen, humanitären Dienst als Alternative zum Dienst an der Waffe zu leisten. Im oben zitierten Eingangssatz des Gründungsmanifestes spiegelte sich das grundsätzliche Dilemma, dem sich die amerikanischen Quäker dabei ausgesetzt sahen. Während viele von ihnen, nicht zuletzt aus der jüngeren Generation, zu den entschiedensten Gegnern des amerikanischen Kriegseintritts zählten, musste es der Führung des AFSC darum gehen, den Eindruck zu zerstreuen, diese Haltung gründe auf einem Mangel an patriotischer Gesinnung. Dieses Spannungsverhältnis prägte die Handlungsweise

1 Gründungsmanifest des Friends National Service Council (so der erste provisorische Name des Gremiums) 30/04/1917, in: AFSCA, AFSC Minutes 1917 (General Meeting).

2 Gründungsmanifest des Friends National Service Council, in: AFSCA, AFSC Minutes 1917 (General Meeting).

3 Die Bestätigung des Namens „AFSC“ erfolgte erfolgte auf einem Treffen am 28. Mai 1917, in: AFSCA, AFSC Minutes 1917 (General Meeting).

Ә OpenAccess. (C) 2022 Daniel Maul, publiziert von De Gruyter. (cc) BY-NC-ND Dieses Werk ist lizenziert unter einer Creative Commons Namensnennung - 4.0 International Lizenz.

https://doi.org/10.1515/9783110675788-004 
und den humanitären Dienst des AFSC bis zum Ende des Krieges. Die Idee eines „constructive service“, mit dem diesem Dilemma begegnet werden konnte, stand deshalb von Anfang an im Mittelpunkt aller Überlegungen.

Vor diesem Hintergrund arbeiteten die Quäker in der Folgezeit eng mit dem Amerikanischen Roten Kreuz zusammen. Es stand als offizielle amerikanische Hilfsagentur an der Spitze einer Entwicklung, in deren Folge die humanitäre Hilfe im Kriegsgebiet als integraler Bestandteil der Kriegführung sowie als „patriotische Bürgerpflicht“ und mithin auch als Mittel der Vergemeinschaftung an der „Heimatfront“ dienen sollte. ${ }^{4}$ Ab Sommer 1917 sollte das AFSC damit nicht nur in wachsender Zahl junge Männer für humanitäre Einsätze im europäischen Frontgebiet ausbilden. Gleichzeitig nahmen die Quäker zu Hause in den USA auch aktiv an einem breiten Diskurs um die Rolle der USA in der Welt teil.

\section{A sense of unity and purpose. Der Aufbau einer Quäkerhilfsorganisation}

In der Gründung des AFSC drückte sich vor allem anderen der Wunsch einer Gruppe von Ostküsten-Quäkern aus, eine Antwort auf die komplexen Herausforderungen $\mathrm{zu}$ finden, vor die der amerikanische Kriegseintritt die Society of Friends in den USA stellte. Am 30. April 1917 fand unter diesen Vorzeichen die oben zitierte Zusammenkunft von 14 Vertreterinnen und Vertretern der wichtigsten Quäkerrichtungen - Orthodoxen und Hicksites - im Whittier Hotel in Philadelphia statt. Initiator des Treffens war Henry Cadbury, Professor für Bibelkunde in Haverford, den Vorsitz hatte Alfred Scattergood inne, ein Bankier aus Philadelphia und bedeutender Vertreter der orthodoxen Glaubensrichtung. Wichtigster Tagesordnungspunkt war der Vorschlag von Rufus Jones - der, wenngleich nicht zugegen, die Rolle des Vorsitzenden des Komitees (chairman) zugetragen bekam -, eine „Emergency Unit at Haverford College“ einzurichten. Ihr Hauptzweck sollte es sein, junge Quäker auf einen humanitären Einsatz in Frankreich vorzubereiten. ${ }^{5}$

\footnotetext{
4 Brandon Little, „An Explosion of New Endeavours: Global Humanitarian Responses to Industrialized War Fare in the First World War Era“, in: First World War Studies 5, 1 (2014), 1-16; Julia Irwin, „The Disaster of War: American Understandings of Catastrophe, Conflict and Relief“, in: First World War Studies 5, 1 (2014), 17-28.

5 Teilnehmer der Sitzung, welche die drei Hauptrichtungen der Quäker in den USA repräsentierten, waren: Henry W. Comfort, Charles J. Rhoads, Alfred G. Scattergood, Anne G. Walton, Stanley R. Yarnall vertraten die Orthodoxen. Arabella Carter, William H. Cooks, Jesse H. Holmes, Lucy Biddle Lewis waren die Vertreter der Friends General Conference, die die Jahresversamm-
} 
Jones' Hauptinspiration waren die britischen Quäker, die seit 1914 eine Friends Ambulance Unit (FAU) unterhielten, in der Quäker-Freiwillige in Frankreich hinter den Frontlinien Sanitätsdienst leisteten. Auch in anderer Hinsicht bot das britische Beispiel Anschauungsmaterial, insbesondere was die Frage der Kriegsdienstverweigerung aus Gewissensgründen und den Aufbau alternativer humanitärer Dienste betraf: Nach Einführung der allgemeinen Wehrpflicht $1916^{6}$ drohten allen, die sich dem Einziehungsbefehl widersetzten, lange Gefängnisstrafen. Die Quäker im Vereinigten Königreich boten Wehrdienstverweigerern vor diesem Hintergrund rechtliche Beratung an - und zwar unabhängig von ihrer Zugehörigkeit zur Society of Friends. Parallel entstand neben der FAU noch das Friends War Victim Relief Committee (FWVRC). Während Erstere sich darauf konzentrierte, Zivilisten an der Frontlinie $\mathrm{zu}$ versorgen, die unmittelbar vom Kampfgeschehen betroffen waren, beschäftigte sich Letzteres insbesondere mit dem Wiederaufbau im Hinterland der Front, errichtete Notunterkünfte und Hospitäler. Dass zwei unterschiedliche Organisationen im Namen der Quäker humanitäre Hilfe leisteten, spiegelte dabei die unterschiedlichen Positionen wider, die in England unter Quäkern hinsichtlich des Krieges und des „richtigen“ humanitären Engagements in Kriegszeiten bestanden. In der FAU sammelten sich all jene, die bereit waren, sich in die offiziellen militärischen Strukturen nach Vorbild des Britischen Roten Kreuzes einzufügen und damit auch einen unmittelbaren Beitrag zum Kriegsaufwand zu leisten. Dagegen vertrat das FWVRC die offizielle „pazifistische“ Linie der Society of Friends: Hilfe für Soldaten ja, aber keine Einordnung in militärische Hierarchien. ${ }^{7}$

lungen der Hicksites bündelte. Homer Morris, Vincent D. Nicholson und L. Hollingsworth Wood vertraten das Five Years Meeting, das als größtes Gremium ebenfalls orthodox war und auch viele evangelikale Gemeinschaften einschloss. Inoffiziell vertreten waren darüber hinaus Henry J. Cadbury und J. Barnard Walton, beide orthodoxe Quäker, Professoren in Haverford und gleichzeitig prominente Mitglieder der radikalpazifistischen Fellowship of Reconciliation. Den besten Überblick über die Gründungsphase des AFSC bietet William J. Frost, „Our Deeds Carry Our Message: The Early History of the American Friends Service Committee.“ Quaker History 81, 1 (1992): 1-51.

6 Großbritannien nahm hier eine Sonderstellung ein, weil das Land als einziges im Konzert der europäischen Mächte keinen verpflichtenden Wehrdienst kannte. Dennoch verfügten die britischen Quäker durch ihre Verbindungen in andere Teile des britischen Empire über Erfahrungen mit dem Problem der Kriegsdienstverweigerung. Namentlich Australien und Neuseeland hatten als Folge des anti-japanisch inspirierten Imperial Defense Plans Lord Kitcheners 1909 und 1910 eine allgemeine Wehrpflicht für junge Männer eingeführt. Greenwood, Friends and Relief, 165193.

7 Diese Unterschiede fanden ihren Niederschlag auch in der Tatsache, dass nur das FWVRC organisatorisch mit dem London Yearly Meeting verbunden war, die FAU dagegen außerhalb der offiziellen Strukturen agierte. Während es sich bei der FAU folglich um eine Sanitätseinheit 
Ein zweiter Zweig der Quäkerhilfe hatte sich bereits Ende 1914 mit der Gründung des Emergency Committee for the Assistance of Germans, Austrians and Hungarians in Distress etabliert. Dieser Ausschuss kümmerte sich um Staatsbürger der Mittelmächte, die sich bei Beginn des Krieges auf dem Gebiet des britischen Empire befanden und als sogenannte enemy aliens in aller Regel ohne Einzelfallprüfung interniert wurden. Von allen Quäkeraktivitäten während des Krieges war diese mit Abstand die unpopulärste. Teile der Presse wandten sich in scharfen Tönen gegen die Hilfe für Zivilinternierte, welche gelegentlich in die Nähe des Landesverrats gerückt wurde. Daran konnte auch die offizielle Anerkennung des Emergency Committee kaum etwas ändern, das von Regierungsseite im War Charities Act von 1916 als gemeinnützig und kriegswichtig eingestuft wurde. ${ }^{8}$

Die Aktivitäten und Nöte der britischen Glaubensgenossen wurden von amerikanischer Seite seit Kriegsbeginn intensiv verfolgt und begleitet. Publikationen der Quäker quer durch alle Zweige der Society of Friends gaben Beiträgen zum Einsatz insbesondere der FAU und des FWVRC breiten Raum. Amerikanische Quäker spendeten Geld und Kleidung und eine kleine Anzahl von jungen Quäkern hatte sich freiwillig für den Dienst in der FAU gemeldet und damit eine direkte und persönliche Verbindung zum Einsatz geschaffen. Dass ein Großteil der Quäker in den USA auf diese Weise mit der Arbeit der britischen Einheiten vertraut war, bot

\footnotetext{
handelte, die sich vorwiegend aus Quäkern zusammensetzte, war das FWVRC ein offizielles Organ der Quäkerhilfe. Greenwood, Friends and Relief, 178-184; allgemein zu den Unterschieden beider Organisationen Kennedy, „Fighting About Peace: The No-Conscription Fellowship and the British Friends Service Committee, 1915-1919“; Martin Ceadel, „The Quaker Peace Testimony and Its Contribution to the British Peace Movement: An Overview“, in: Quaker Studies 7, 1 (2002), 9-29. 8 Die große Zahl der Zivilinternierten stellte die britische Regierung, ganz ähnlich wie die deutsche und die französische Regierung, vor ein doppeltes Problem: Zunächst stellten die „feindlichen Ausländer“, deren Zahl auf beiden Seiten der Front in die Tausende ging, ein Versorgungsproblem dar. Hier kam der Arbeit der Quäker, die für einen Teil der Kosten aufkamen, eine bedeutende Funktion zu. Zudem stellte die Versorgung der Zivilinternierten für alle Seiten eine Art gegenseitige Garantie für die jeweils eigenen im Feindesland internierten Staatsangehörigen dar. Schließlich stellten das Emergency Committee und sein Pendant auf deutscher Seite, die Hilfsstelle für Deutsche im Ausland und Ausländer in Deutschland, die einen regen Austausch miteinander pflegten, einen wichtigen inoffiziellen Kommunikationskanal sowohl für die betroffenen Personen als auch für offizielle Stellen dar. In diesem Umstand liegt die wahrscheinlichste Erklärung, warum die Tätigkeit dieser Gruppen zu keinem Zeitpunkt grundsätzlich in Frage gestellt wurde. Zum Problem der Zivilinternierten im Ersten Weltkrieg Stibbe, Civilian Internment During the First World War: A European and Global History, 1914-1920; Proctor, Civilians in a World at War, 203-238.
} 
dem AFSC einen Startvorteil und eine Art roten Faden für das Vorgehen während der ersten Wochen nach dem Kriegseintritt. ${ }^{9}$

Gleichzeitig eignete sich die britische Erfahrung jedoch nur bedingt als Blaupause für die US-Quäker, deren Situation zum Zeitpunkt des Kriegseintritts sich in wesentlichen Punkten von der ihrer britischen Glaubensgenossen unterschied. So war die Frage der Kriegsdienstverweigerung aus Sicht der Quäker in den USA weniger existenziell, denn die Angehörigen der „historischen Friedenskirchen“, zu denen neben den Quäkern die Mennoniten und Herrnhuter Brüder (Brethren) zählten, konnten nach Gesetzeslage allein wegen ihrer Mitgliedschaft in der betreffenden Glaubensgemeinschaft mit einer Rückstellung vom Armeedienst rechnen. ${ }^{10}$ Den Initiatoren des AFSC musste es deshalb darum gehen, eine Antwort auf die spezifischen Herausforderungen zu geben, vor die sich die Society of Friends in den USA zum Zeitpunkt des Kriegseintritts gestellt sah. „To assist all Friends in America in making an adequate contribution to the needs of the hour (....) - so lautete der selbstgewählte Auftrag des AFSC in seiner ersten Stellungnahme an alle Quäkergemeinden im Land. ${ }^{11}$

Ein primäres Ziel war dabei, „unity of purpose and effort“ innerhalb der Society of Friends in den USA herzustellen. Angesichts der tiefen theologischen und kulturellen Gräben, welche die verschiedenen Richtungen der Quäker - Orthodoxe, Hicksites, Liberale, Evangelikale oder „programmed friends“ - voneinander trennten, nahm der Appell an die Einheit eine zentrale Bedeutung ein. Dabei ist auffällig, dass die Initiative zur Gründung des AFSC maßgeblich von Quäkern getragen wurde, die im Young Friends Movement sozialisiert waren. Bei der Gründung des AFSC hatten diese einen Startvorteil, weil vor allem sie es waren, die vor 1917 die scharf gezogenen Grenzen zwischen den einzelnen Quäkerrichtungen punktuell überwinden konnten. Zudem verfügte das YFM auch über einen Vorsprung hinsichtlich der Zusammenarbeit mit Quäkern in anderen Ländern - insbesondere in England und dem britischen Commonwealth, wo führende Köpfe der Quäkerhilfsorganisationen dem YFM entstammten. Der Einfluss des YFM, oder zumindest seiner liberalen Vertreter, war denn auch allein durch die Verwendung des Begriffs Service im Namen des AFSC deutlich erkennbar. Service signalisierte Diesseitigkeit und Weltzugewandheit und stand in einem freilich selten offen erklärten Gegensatz zum evangelikalen Anspruch, Seelen zu retten. ${ }^{12}$

9 Friends Reconstruction and Relief Work in Europe, in: AFSC-Bulletin 3 (1917); eine Zusammenfassung im Rückblick: A new Birth of Quakerism in England, in: AFSC-Bulletin 6 (1917).

10 Mennonite Cooperation, in: AFSC-Bulletin 21 (1918).

11 Suggestions for Local Organization, in: AFSC-Bulletin 1 (1917).

12 Siehe auch Kapitel 1. 
Ein wesentlicher Faktor für den Erfolg des AFSC war, dass sich hinter dem Anspruch, jungen Quäkern einen Weg zu öffnen, ihrem Glauben zu folgen und sich dem Dienst an der Waffe zu verweigern, zumindest im Prinzip alle Richtungen versammeln konnten. Dies galt sogar im Lichte dessen, dass die Haltungen zum Kriegseintritt der USA innerhalb der Society of Friends weit auseinandergingen. Radikalpazifistische Positionen repräsentierten dabei allenfalls die Überzeugungen einer Minderheit der US-amerikanischen Quäker. Unter dem Einfluss des YFM hatte eine absolute Auslegung des peace testimony als Teil der religiösen Identität der Quäker zwar an Stärke gewonnen, war jedoch weit von einer Hegemonialstellung entfernt. ${ }^{13}$

Die meisten Friends pflegten ein durchaus pragmatisches Verhältnis zum peace testimony. Und während eine Mehrheit der Quäker - wie eingangs beschrieben - wohl in den ersten Kriegsjahren aus der amerikanischen Beobachterposition einer Politik der Nicht-Einmischung der USA in den Krieg befürwortete, schuf der Kriegseintritt eine neue Situation, in der sich die Haltung der Quäker nur wenig von der des liberalen protestantischen Mainstreams unterschied. Hier vollzog sich nach dem April 1917 ein deutlicher Schwenk hin zur Unterstützung der amerikanischen Kriegführung. ${ }^{14}$ Danach sahen sich jene Friends, die sich der Kriegsbeteiligung weiterhin entgegenstellten, in der Öffentlichkeit zunehmend Kritik und zuweilen offener Feindseligkeit ausgesetzt. Diese Stimmung machte selbst vor Quäkereinrichtungen keinen Halt. Ein prominentes Opfer war Henry Cadbury, der sich aufgrund seiner öffentlich geäußerten Kritik an der aufgeheizten Kriegsstimmung aus seiner Lehrposition in Haverford gedrängt sah. Auch die Reaktionen junger wehrfähiger Quäker zum Kriegseintritt variierten deutlich: Von totaler Verweigerung bis zu freiwilliger Meldung zum Militärdienst

13 Christina Bowen, The Fighting Quakers: A New Vision for the Peace Testimony During World War I, 2019, (Diss., Haverford College, Haverford); Bishop und Jung, „Seeking Peace: Quakers Respond to War“; Ceadel, „The Quaker Peace Testimony and Its Contribution to the British Peace Movement: An Overview“.

14 Richard M. Gamble, The War for Righteousness : Progressive Christianity, the Great War, and the Rise of the Messianic Nation (Wilmington, Del.: ISI Books, 2003). Für einen Überblick Jonathan Ebel, „Religion (USA)“, in: Ute Daniel, Peter Gatrell, Oliver Janz, Heather Jones, Jennifer Keene, Alan Kramer, and Bill Nasson (Hg.), 1914-1918-online. International Encyclopedia of the First World War (Freie Universität Berlin, Berlin, 2014-10 - 08) (Religion (USA) | International Encyclopedia of the First World War (WW1) (1914-1918-online.net) Zugriff: 9.3.2021); Jonathan Hansen, The Lost Promise of Patriotism: Debating American Identity, 1890-1920 (Chicago: University of Chicago Press, 2010), 158. 
waren alle Positionen vertreten. Vor allem in den Gemeinden im Mittleren Westen spürten junge Männer den Druck der öffentlichen Meinung. ${ }^{15}$

Gerade mit Blick auf diese Gruppe galt es aus Sicht des AFSC, Wege aufzuzeigen, dem Druck standzuhalten. Dieser Ausweg, darüber bestand früh Einigkeit, war nur dann gangbar, wenn es gelang, jungen Quäkern eine echte Alternative zum Dienst an der Waffe anzubieten. Der entscheidende Punkt war, dass die Alternative eine Gewissensentscheidung gegen den Kriegsdienst nicht als Ausdruck einer unpatriotischen Gesinnung erscheinen lassen durfte. Dieser Anspruch zeigte sich im Gründungsmanifest des AFSC und dessen Selbstverpflichtung zum ,constructive service“.Diese richtete sich somit sowohl nach außen - an eine potenziell feindselige Öffentlichkeit - als auch nach innen, in die Quäkergemeinden selbst. Sie gab jenen Friends eine Hilfestellung, die um die Grundfesten des Quäkertums und seiner pazifistischen Überzeugungen fürchteten, und versuchte gleichzeitig auch jene ins Boot $\mathrm{zu}$ holen, die den Kriegseintritt befürworteten. Die Betonung des „konstruktiven“ und patriotischen Charakters des vorgesehenen Ersatzdienstes als einer Alternative sowohl zum militärischen Fronteinsatz als auch zu einem absolut gesetzten Pazifismus stellte eine unabdingbare Voraussetzung dar, um Einheit innerhalb der Society of Friends herzustellen und aufrechtzuerhalten. Es ging darum, einen Ersatzdienst zu schaffen, der sowohl von der Öffentlichkeit als auch von der eigenen Klientel als „constructive“ und loyal gegenüber der eigenen Regierung anerkannt wurde.

Um dies zu gewährleisten, mussten einige Voraussetzungen erfüllt sein: Zunächst musste der Einsatz dort stattfinden, wo er - was Gefahr und Anstrengung betraf - dem militärischen Dienst vergleichbar war. Es konnte sich folglich nur um einen Dienst handeln, der am Kriegsschauplatz und nahe der Frontlinie geleistet wurde. Dem Vorwurf der Illoyalität konnten die Quäker nur dann entgegentreten, wenn sie Bereitschaft „for the quality of sacrifice others are rendering“ zeigten. ${ }^{16}$ Nur wenn der Dienst darüber hinaus gleichzeitig als Ausdruck einer generellen Weltsicht erschien, konnte der Eindruck eines bequemen Rückzugs und mithin des „Drückebergertums“ verwischt werden. Die Quäker mussten durch ihren Dienst ihre Opferbereitschaft demonstrieren, und durften dabei denen, die ihren Dienst am Vaterland mit der Waffe leisteten, in nichts nachstehen. Gleichzeitig musste er jenen als Vorbild und Anlaufstelle dienen, die ohne die komfortable Sicherheit der Quäker zu denselben Schlüssen gelangt waren. Ruhe man sich alleine auf dem eigenen Recht auf Kriegsdienstverweigerung aus, ohne einen

15 Eine Vielzahl von Material das die Situation dokumentiert findet sich etwa im Folder AFSCA, General Administration 1917 (Young Friends Movement).

16 Service in Financial Contribution, in: AFSC Bulletin 2 (1917). 
konstruktiven Beitrag zu leisten, so die Linie des AFSC, „we shall have sold our birth-right. We shall have lost all right to speak. We shall have destroyed on the part of others all willingness to listen. "17 In diesem Sinn betrachtete das AFSC die zwei Säulen seiner Tätigkeit, die Beratung von Kriegsdienstverweigerern - aus religiösen und nichtreligiösen Motiven ${ }^{18}$ - sowie die Suche nach praktischen Alternativen zum Kriegsdienst, nicht als Gegensätze, sondern als zwei Seiten einer Medaille.

Deshalb bemühte sich das AFSC ab Mai 1917 um das Einverständnis der Regierung, den Quäkern den Aufbau eines alternativen Dienstes im Kriegsgebiet zu gestatten. Dabei kamen Kontakte zum Tragen, welche die Quäker mit progressiven und sozialreformerischen Vertretern innerhalb der Wilson-Administration pflegten. Das AFSC signalisierte dabei von Anfang an, dass es trotz seiner grundsätzlich pazifistischen Haltung keineswegs in radikaler Opposition zur Außenpolitik der Regierung stand. Vielmehr propagierte es den eigenen Dienst als Antwort auf die gestiegene globale Verantwortung der USA: „As the world obligation of the US has begun to assume gigantic proportions“, hatte es unmittelbar nach der Gründung des AFSC geheißen, mussten und wollten auch die Quäker ihren Beitrag leisten. ${ }^{19}$ Die ersten Signale aus Washington waren dann auch durchaus hoffnungsvoll. Präsident Wilson selbst äußerte in einem Schreiben an Rufus Jones im August 1917 „deep appreciation of the reconstruction work proposed“. Diese Aussage bezog sich allerdings nur auf Personen außerhalb des wehrfähigen Alters sowie auf Freiwillige, die bislang noch nicht eingezogen worden waren - und auf Letztere lediglich unter dem Vorbehalt, dass diese bei Einberufung ihren Hilfsdienst beendeten. ${ }^{20}$

Es war der doppelte Anspruch auf constructive service auf dem Kriegsschauplatz und die umfassende Vertretung von Gewissensverweigerern, der sich schwer

17 Service in Financial Contribution, in: AFSC Bulletin 2 (1917).

18 Das AFSC trat anfänglich auch als Vertretung von religiösen und säkularen Gewissensverweigerern auf, die etwa aus dem Umfeld des Fellowship of Reconciliation, der Methodisten und anderer Organisationen kamen.

19 Friends Reconstruction and Relief Work in Europe, in: AFSC-Bulletin 3 (1917). Rufus Jones hatte sich zuvor mit einer Empfehlung des Roten Kreuzes an Woodrow Wilson mit der Bitte um eine Entscheidung gewandt ,whether this important piece of Red Cross Work is satisfactory alternative service for members of our religious Society, and (2) whether permits may be granted for these men of the Unit, without the delay of going to mobilization camps, to accept the call to go to France Sept. $1^{\text {st }}$. Rufus Jones and Woodrow Wilson 15/8/1917 in: AFSCA, General Administration 1917 (US Government, Office of President).

20 Woodrow Wilson an Rufus Jones 28/8/1917, in: AFSCA, General Administration 1917 (US Government, Office of President). 
mit den Zielen der Regierung und noch weniger mit denen des Militärs in Einklang bringen ließ.

Die eigentliche Sollbruchstelle bestand dabei im Einsatz von Kriegsdienstverweigerern in Frontnähe. Für solche Personen, die aus religiöser Überzeugung den Dienst an der Waffe verweigerten, wollte man von offizieller Seite lieber Möglichkeiten in den USA selbst schaffen, etwa bei Aufforstungsprojekten oder in der Landwirtschaft. Denn was aus Sicht des Militärs vermieden werden sollte, war, dass die Gewissensverweigerer im Kontakt mit der kämpfenden Truppe ihre „subversive“ Kraft entfalten konnten. ${ }^{21}$ Die Auseinandersetzung der ersten Monate drehte sich unter diesen Vorzeichen nicht in erster Linie um das „Ob“, sondern vor allem um das „Wer“ und das „Wie“ des Einsatzes.

Ungeachtet dieser Unklarheiten galt es in den ersten Monaten nach Kriegsbeginn, das AFSC und seine Position unter den Quäkern im Land bekannt zu machen, „to rally all friends in America (...) behind a great programme of service“. Besonderes Augenmerk galt dabei vor allem jenen Gemeinden außerhalb des unmittelbaren Einflussbereichs des Komitees an der Ostküste. „Since the problem of the hour are peculiarly young peoples problems“, übernahm dabei das Young Friends Movement eine entscheidende Rolle. Seine Verankerung quer zu allen Ausrichtungen der Quäker bis in die evangelikalen Gemeinden des Five Years Meeting im Mittleren Westen war ein zusätzliches Plus. ${ }^{22}$ In der Folge wurden gezielt young friends angesprochen, die durch Vortragsreisen und Versammlungen im ganzen Land dem AFSC die nötige Aufmerksamkeit verschaffen sollten.

Die Berichte dieser „Missionare“ des AFSC geben einen guten Eindruck von der Größe der Aufgabe und der Vielfalt der Haltungen und Erwartungen an das Komitee zu diesem Zeitpunkt. Ein Beispiel war Thomas „Tom“ Jones aus Indiana, der im Sommer 1917 Gemeinden im Mittleren Westen bereiste. Als Vorbereitung hatte Jones im Juni - vermittelt von den Gemeinden des Five Years Meeting - einen persönlich gehaltenen Brief an alle Männer im wehrfähigen Alter geschrieben. Darin ermutigte (encouraged) er junge Männer, seinem eigenen Beispiel zu folgen und ihr Recht auf Verweigerung als Quäker wahrzunehmen. Im Wesentlichen gab er den Rekruten Argumente an die Hand, um dem Vorwurf zu entkommen, „Slackers“ (Drückeberger) zu sein. Durch die Art von „constructive service“, die das AFSC vertrat, könnten sich Quäker jenseits ihres anerkannten Rechts als Angehörige einer „peace church“ auf einer Stufe mit anderen, etwa „research chemists or statisticians“ sehen, deren zivilen Dienst die Regierung als „as im-

21 AFSCA, General Administration 1917 (War Department, US Government).

22 Vincent Nicholson an Walter Woodward (Five Years Meeting) 7/6/1917, in: AFSCA, AFSC General Administration (Five Years Meeting, Young Friends 1917). 
portant as fighting“ anerkenne. Man erhalte durch die Regierung die Gelegenheit, einen Beitrag zu leisten, der im Einklang mit dem Glauben und zugleich im Interesse der Nation sei. Constructive service im Sinne des AFSC signalisiere, „that Friends are willing to lay down their lives, if need be, for their principles, just as soldiers who give their lives for their principles“. Die Regierung erkenne zunehmend an, schloss Jones optimistisch, dass das Opfer, das die Quäker mit diesem Dienst erbrächten, ebenso wie der Dienst an der Waffe „of utmost value to the life and freedom of our nation“ sei. ${ }^{23}$ Jenseits dieser Einschätzungen geben die Berichte von Jones' folgender Vortragsreise durch die Quäkergemeinden Ohios und Indianas ein lebendiges und vielfältiges Bild der Haltungen, der gewaltigen Erwartungen, aber auch der Vorbehalte, mit denen die Arbeit des AFSC zu kämpfen hatte. Jones’ Vorträge, die zuweilen in Gemeindehäusern und an Bahnhöfen gehalten wurden, folgten einem festen Schema, deren Hauptüberschriften den Grundton klar erkennen lassen: „The Friends Principles for which we are willing to die“, „The especial message of the Reconstruction work to our Society at this time and our absolute obligation to sacrifice our all for it“; „The courses of action that lie open to a real conscientious objector at this time“. Es ging folglich um „echte“ Gewissensverweigerung sowie um „echte“, „kompromisslose“ und „äußerste“ Opferbereitschaft. Nach Jones’ Überzeugung und Erfahrung war dies eine Botschaft „for which people are waiting everywhere“. Vielerorts fand er die Verzweiflung der Menschen aufgrund des Drucks, den die lokalen Rekrutierungsbehörden auf die Gemeinden ausübten, so groß, „that they grasp for our message like a drowning man for a straw“. Nicht alle konnte er allerdings überzeugen. Immer wieder erhoben sich Stimmen, welche die Position des AFSC in die Nähe des Landesverrats rückten. Beispielhaft war der Einwurf eines älteren (orthodoxen) Quäkers bei einer Versammlung in Waynesville, Ohio. Dieser wurde ungehalten, als Jones ausführte, dass Quäker keine Drückeberger seien, wenn sie sich zu kämpfen weigerten, „provided they had a program of love for which they were willing to lay down their lives“. „This is no time to preach love“, habe ihm der Mann mit großer Wut und dem Verweis auf die deutschen Greueltaten in Belgien entgegengeschrien („What would you have done if you had been in Belgium?“). In der Regel waren diese Stimmen laut Jones, wenngleich überall anzutreffen, doch in der Minderheit gegenüber denen, die dankbar die Hilfestellung des AFSC im Umgang mit dem Drückeberger-Vorwurf aufnahmen. ${ }^{24}$

23 Thomas Jones an Walter Woodward (Five Years Meeting) 24/7/1917, in: AFSCA, AFSC General Administration 1917 (Five Years Meeting, Young Friends).

24 Thomas Jones an Vincent Nicholson 13/9/1917, in: AFSCA, AFSC General Administration 1917 (Five Years Meeting, Young Friends). 


\section{Das AFSC in Frankreich}

Vor diesem Hintergrund schuf das AFSC ab Mitte Mai Strukturen, die es den Quäkern erlaubten, eine erste Gruppe von relief workers für den Einsatz im europäischen Kriegsgebiet auszubilden. Im Mai erfolgte die Einrichtung eines Sekretariats, dessen erster Vorsitzender (Executive Secretary, im Folgenden: Generalsekretär) Vincent „Tim“ Nicholson, ein Anwalt aus Indiana, wurde. ${ }^{25}$ Den Vorsitz des Finanzkomitees, das für die Sammlung von Spendengeldern zuständig war, übernahm Alfred Scattergood, der über ein Budget von zunächst 150.000 USDollar verfügte. Für die Beratung und alle anderen Aktivitäten zugunsten von Kriegsdienstverweigerern wurde ein Exemption Committee unter William B. Harvey, eines weiteren orthodoxern Quäkers, eingerichtet. Parallel schufen die Quäker ein Aufsichtsgremium (Executive Board), in das die verschiedenen Quäkerrichtungen jeweils ihre Vertreter schickten und zu dessen ersten Vorsitzenden Henry Cadbury und Rufus Jones berufen wurden. Als Hauptquartier fungierte ab Juni das meeting house der (orthodoxen) Quäkergemeinde in der 20 South $12^{\text {th }}$ Street im Zentrum von Philadelphia. Die große Bedeutung, die der Vermittlungsarbeit insbesondere gegenüber einer potenziell feindseligen öffentlichen Meinung eingeräumt wurde, spiegelte sich in der Einrichtung eines eigenen Publicity Department, das bereits im September seine Arbeit aufnahm, als die ersten Quäkerhelfer nach Europa geschickt wurden. ${ }^{26}$

Das Herzstück der Vorbereitung war ein Ausbildungslager für zunächst 100 angehende Helfer, das im Juli 1917 auf dem wenige Kilometer außerhalb von Philadelphia gelegenen Gelände von Haverford College eröffnete. Die Teilnehmer, zunächst ausschließlich Männer, erhielten hier ein sechswöchiges Training, das neben Einweisungen in Erster Hilfe, Sozialarbeit und medizinischem Grundwissen auch Sprachkurse und landeskundliche Vorlesungen umfasste, die ihnen

25 Nicholsons Position war vor allem mit Blick auf die Außendarstellung des AFSC besetzt worden, er verfügte dagegen kaum über organisatorische Erfahrungen, was ihm in den folgenden Monaten häufiger Kritik aus dem AFSC einbrachte. So störten sich einige der Mitglieder des Komitees daran, dass Nicholson der Planung einer ausgedehnten Europareise im Oktober (die nicht zustande kam) höheres Gewicht beigemessen hatte als der Reorganisation des AFSC zu Hause. Für Morris Leeds, Mitglied des AFSC, war das „convincing proof, if proof were needed, that he does not think adequately along lines of organization and administration to carry out his plans“. Morris Leeds an Rufus Jones (Kopie an Alfred Scattergood und Henry Cadbury) 9/10/1917, in: AFSCA, AFSC General Administration 1917.

26 Zusätzlich wurden mit dem zum Schatzmeister berufenen Charles Jenkins sowie den Unternehmern Henry Tatnall Brown und Morris Leeds drei weitere Mitglieder aufgenommen. Reports of the Subcommittee on Reorganisation of the General Committee 11/5/1917, in: AFSCA, AFSC General Administration 1917; Meeting 19/5/1917, in: AFSCA, AFSC Minutes 1917 (General Meeting). 
Kenntnisse über Politik und Kultur des Einsatzgebiets vermitteln sollten. Ein großes Gewicht wurde auch auf die Ausbildung praktischer Fertigkeiten gelegt. Haverford unterhielt dafür eine eigene Landwirtschaftsschule samt Modellfarm im nahe gelegenen Westtown, eine Tischlerei und eine Autowerkstatt. ${ }^{27}$

Alle Vorbereitungen für einen humanitären Einsatz konzentrierten sich von Anfang an auf Frankreich. Indem man einem Land half, das mit den USA verbündet war, ließ sich in der Öffentlichkeit am besten dem Eindruck begegnen, die Antikriegsposition der Friends sei gleichbedeutend mit einer neutralen Haltung im Krieg. Ein Einsatz in Frankreich sprach somit auch jene Quäker an, für die ein alternativer Dienst nur dann in Frage kam, wenn er sie nicht dem Verdacht einer unpatriotischen Haltung aussetzte. Um Zugang zum Kriegsschauplatz in Frankreich zu erhalten, musste man aber zunächst die Unterstützung des Amerikanischen Roten Kreuzes gewinnen, dem seit 1914 die Verantwortung für sämtliche zivile humanitäre Hilfsaktivitäten in den europäischen Kriegsgebieten oblag. ${ }^{28}$

Kraft dieses Monopols, das auf einen präsidentiellen Beschluss zurückging, und seiner privilegierten Beziehungen zum US-Militär, regelte das ARC vom Zeitpunkt des Kriegseintritts an den Zugang anderer humanitärer Organisationen zum europäischen Kriegsschauplatz. Dadurch oblag es dem ARC auch zu definieren, was als gleichzeitig humanitär und im nationalen Interesse gelten sollte. Gleichzeitig wuchs das ARC innerhalb kurzer Zeit zu einer Massenorganisation, die auf dem Höhepunkt 1919 ca. 20 Millionen Mitglieder zählte - ein knappes Drittel der erwachsenen amerikanischen Bevölkerung. ${ }^{29}$ In dieser Position war

27 J. William Frost, „Our Deeds Carry Our Message: The Early History of the American Friends Service Committee“, in: Quaker History 81, 1 (1992), $16 \mathrm{f}$.

28 Siehe zur Diskussion um die Hilfe in Frankreich Michael McGuire, „A Highly Succesful Experiment in International Partnership: The Limited Resonance of the American Committee for Devastated France“, in: First World War Studies 5, 1 (2014), 101-115.

29 Dabei nahm das 1881 gegründete ARC nicht erst im Krieg eine Sonderrolle innerhalb der internationalen Rotkreuzfamilie ein. Früher als andere nationale Rotkreuzgesellschaften hatte das ARC das ursprünglich auf die Versorgung verwundeter Soldaten in Kriegszeiten begrenzte Mandat der Genfer Konventionen um eine zivile Dimension erweitert. Neben Naturkatastrophen im eigenen Land, wie etwa dem großen Erdbeben von San Francisco von 1906, wurde das ARC früh auch über die USA hinaus im Falle von Naturkatastrophen tätig, wie dem großen Seebeben von Messina. Bereits 1908 richtete das ARC am Stammsitz in Washington ein international relief bureau zur Koordination seiner internationalen Einsätze ein. Amerikanische Politiker begriffen im Zeitalter wachsenden globalen Einflusses der USA das ARC zunehmend als ein diplomatisches Instrument. Bereits unter den Präsidentschaften von Theodore Roosevelt, Howard Taft und Woodrow Wilson wurde das ARC sukzessive zur „official voluntary relief organisation of the United States“. Irwin, Making the World Safe: The American Red Cross and a Nation's Humanitarian Awakening, 13-104. 
das Rote Kreuz federführend, den zivilen Einsatz in Frankreich an der Seite des Militärs als integralen Bestandteil des nationalen Kriegsaufwandes zu propagieren. Es etablierte dabei ein humanitäres Ethos, das die zivile Hilfe in den europäischen Bündnisstaaten als patriotischen Akt und „American obligation“ definierte. Auch die diplomatische Funktion amerikanischer Hilfe wurde betont, indem sie die soziale Ordnung und den Durchhaltewillen in den Empfängerländern stärke, zur politischen und wirtschaftlichen Stabilität beitrage und gleichzeitig ein positives Bild der USA nach außen projiziere. ${ }^{30}$ Damit erstreckte sich der Einfluss des ARC - über seine Rolle als gatekeeper für den Zugang nach Frankreich hinaus - in vielfacher Hinsicht auf die humanitäre Arbeit der Quäker.

Mit Hinblick auf Frankreich kam dem AFSC anfänglich zugute, dass einige seiner Mitglieder enge Kontakte in die Führungsspitze des Amerikanischen Roten Kreuzes pflegten. Grayson Murphy etwa, Chef des Amerikanischen Roten Kreuzes in Frankreich, war Haverford-Absolvent und Rufus Jones freundschaftlich verbunden. Auch Homer Folks, von Frühjahr 1917 an Direktor für zivile Angelegenheiten des ARC in Frankreich, war mit der Arbeit der Quäker vertraut. In einem Schreiben an den Kriegsminister Newton Baker rühmte Folks die Arbeit der Briten als „finest relief work that I have ever seen in my life“ und bat, die AFSC-Männer einsetzen zu dürfen, die „highly qualified and equipped for this important service“ seien. ${ }^{31}$ Von beiden erfuhr das AFSC Unterstützung in seinem Anliegen.

Bereits im Frühsommer 1917 lud Murphy zwei Mitglieder des AFSC, J. Henry Scattergood und L. Hollingsworth Wood, nach Frankreich ein, um die Einsatzmöglichkeiten für eine amerikanische Quäkereinheit vor Ort auszuloten. ${ }^{32}$ Wood und Scattergood bereisten das Kriegsgebiet und stellten auch engere Kontakte zwischen der ARC-Mission in Paris und den bereits im Land tätigen britischen reconstruction units her. ${ }^{33}$ Die Briten leisteten nach Überzeugung von Folks vor allem beim Bau von Notunterkünften und im medizinischen Bereich Pionierarbeit, von der das ARC vieles lernen könne. ${ }^{34}$ Eine Zusammenarbeit mit dem AFSC war aus Sicht des ARC aber auch aus einem weiteren Grund attraktiv: Unter den

30 Irwin, Making the World Safe: The American Red Cross and a Nation's Humanitarian Awakening, 67-104.

31 Red Cross War Council an Newton Baker 31/8/1917, in: AFSCA, AFSC General Administration 1917 (American Red Cross, letters).

32 Grayson Murphy an Vincent Nicholson 28/5/1917, in: AFSCA, AFSC General Administration 1917 (American Red Cross, letters).

33 Auf die hohe Reputation der Briten verweisen AFSC-Bulletin 6 (1917) und AFSC-Bulletin 8 (1917). Siehe auch die Korrespondenz zwischen Grayson Murphy (ARC) und Henry Scattergood 6/ 8/1917 und 7/8/1917, in: AFSCA, AFSC General Administration 1917 (American Red Cross; letters). 34 Lester M. Jones, Quakers in Action: Recent Humanitarian and Reform Activities of the American Quakers (New York: Macmillan, 1929)., 22-25. 
Freiwilligen, die das AFSC in Haverford ausbildete, befanden sich College-Absolventen, Ärzte, Ingenieure und andere Spezialisten, an denen es der civilian branch des ARC zu diesem Zeitpunkt mangelte. Dem ARC war nach Einschätzung Scattergoods an einer engen Zusammenarbeit gelegen, weil „our Unit offers it the services of just the class of trained men that it now greatly needs and has not as yet arranged to get from other sources“. ${ }^{35}$ Grayson Murphy bat die Quäker vor diesem Hintergrund darum, weitere Freiwillige für die Ausbildung in Haverford anzuwerben, da er in Frankreich einen immensen Bedarf voraussah. ${ }^{36}$

Im Sommer 1917 schlossen ARC und AFSC eine Vereinbarung, die es den Quäkern erlaubte, die amerikanischen Helfer den bestehenden Wiederaufbaueinheiten der Briten zuzuteilen, gleichzeitig jedoch offiziell als American Friends Unit of the Red Cross aufzutreten. Die AFSC-Rekruten wurden der zivilen Abteilung des ARC in Frankreich zugeordnet, im Hauptquartier des ARC in Paris wurde ein Bureau of Friends Unit of the Department of Civil Affairs eröffnet. Letzteres diente fortan als Koordinationszentrum der gleichzeitig eingerichteten Mission AngloAmericaine de la Société des Amis. ${ }^{37}$ Die amerikanischen Quäker sollten der Kontrolle des ARC unterstehen, für die Kosten von Transfer, Unterhalt und Einsatz der Quäkerhelfer wollte das AFSC vollständig aufkommen. ${ }^{38}$ Das AFSC verpflichtete sich, ,to furnish to the Red Cross men and women who will be thoroughly loyal to your organization, willing to accept without self-seeking, any work to which Friends may properly be called, accentuated by religious motives and high ideals of service“. ${ }^{39}$ Gleichzeitig wurde das AFSC in den USA in ein im Februar 1917 eingerichtetes Committee on Cooperation eingegliedert, das dem ARC dazu diente, die Arbeit aller auf dem Kriegsschauplatz tätigen amerikanischen Hilfsorganisationen zu koordinieren. ${ }^{40}$

Damit war die Grundlage für eine im Wesentlichen reibungsfreie Zusammenarbeit zwischen AFSC und ARC über die Dauer des Kriegseinsatzes geschaffen. Das Rote Kreuz schätzte die Quäker, was sich in wiederkehrenden Bitten um eine Aufstockung der zur Verfügung gestellten Helfer spiegelte. ${ }^{41}$ Als die civilian

35 Concluding Report of our Mission send to France, in: AFSC-Bulletin 6 (1917).

36 Concluding Report of our Mission send to France, in: AFSC-Bulletin 6 (1917).

37 Concluding Report of our Mission send to France, in: AFSC-Bulletin 6 (1917).

38 Alfred Scattergood an Grayson Murphy 6/8/1917; Vincent Nicholson an Grayson Murphy 24/8/ 1917, in: AFSC General Administration 1917 (American Red Cross, letters).

39 Concluding Report of our Mission sent to France, in: AFSC-Bulletin 6 (1917).

40 Das Komitee war im Februar 1917 in Vorbereitung auf den Krieg mit den Spitzen der anderen Relieforganisationen, von Herbert Hoover (Commission for Relief in Belgium), John R. Mott (YMCA) und Cleveland Dodge (Near East Relief) eingerichtet worden; Irwin, Making the World Safe: The American Red Cross and a Nation's Humanitarian Awakening, 73.

41 Concluding Report of our Mission send to France, in: AFSC-Bulletin 6 (1917). 
branch im Sommer 1918 schließlich wie die anderen Abteilungen des Roten Kreuzes unter die Oberaufsicht der Armee gestellt wurde, führte das Rote Kreuz die Verhandlungen mit dem Militär, die sicherstellten, dass AFSC-Personal hierdurch nicht unter die Militärgerichtsbarkeit fiel. Vor diesem Hintergrund fiel es den Quäkern leicht, sich als „organischer Teil“ der Rotkreuzmission zu begreifen. Dank der Zusammenarbeit mit dem Roten Kreuz konnte somit das eigene Handeln nach innen wie nach außen erfolgreich als „constructive service“ dargestellt werden.

Im September 1917 kamen die ersten 54 amerikanischen Quäkerhelfer in Frankreich an. Der größere Teil arbeitete in den folgenden 13 Monaten bis zum Ende des Krieges in reconstruction units hinter den Frontlinien, insbesondere in den Gebieten um die Aisne, Somme und Oise, die vom Kriegsgeschehen verwüstet worden waren. Ihre Hauptaufgabe bestand darin, Unterkünfte für Rückkehrer zu schaffen, wobei meistens Fertighäuser zur Anwendung kamen, deren Bausätze in zwei eigens eingerichteten Produktionsstätten in Dôle und Ornans im französischen Jura vorfabriziert wurden. ${ }^{42}$

Ein zweiter Teil der Quäkerhelfer arbeitete beim Wiederaufbau der Landwirtschaft, insbesondere dort, wo aufgrund des Ausfalls der männlichen Bevölkerung Hilfe benötigt wurde. Die britischen Quäker hatten in Sermaize an der Marne ein Landwirtschaftszentrum mit 150 Mähmaschinen und einer Reihe von anderen Geräten eingerichtet. Nach der Ankunft der AFSC-Helfer wurden weitere Zentren in Golancourt und Gruny an der Somme errichtet, die Saatgut, Kaninchen, Ziegen und Hühner an die lokale Bevölkerung verteilten. ${ }^{43}$

Eine dritte Gruppe von medizinisch geschultem Personal unterstützte ebenfalls bereits bestehende britischen Einrichtungen, wie etwa eine Geburtsklinik in Châlons-sur-Marne oder Erholungsheime für Flüchtlinge aus den Kriegszonen sowie für Rückkehrer. In Sermaize bauten die Quäker unter der Leitung von James Babbitt, einem Arzt aus Philadelphia, ein von den Briten errichtetes allgemeines Krankenhaus aus, in dem vor allem Kriegsopfer behandelt wurden und in dem bis zum Kriegsende ca. 1200 Operationen durchgeführt wurden. ${ }^{44}$

Neben diesen längerfristig angelegten Tätigkeiten bestand ein großer Teil der geleisteten Arbeit in der Linderung akuter Notlagen: So waren „social work of all

42 Six Months of Friends' War Relief Service, in: AFSC-Bulletin 8 (1917).

43 Six Months of Friends' War Relief Service, in: AFSC-Bulletin 8 (1917).

44 Die Ankunft der Amerikaner erlaubte zudem den Aufbau einer Tuberkulosestation im französischen Jura. Im Juni 1918 leisteten die meisten der Quäker in Frankreich Wiederaufbauarbeit, gefolgt von medizinischen Tätigkeiten und der Landwirtschaft. Bericht von Charles Evans über französische Arbeit mit vollständiger Liste der Tätigkeiten n.n., in: AFSCA, General Administration 1918. 
kinds“, die Verteilung von Lebensmitteln und Kleidern, aber auch die Einrichtung von Möbellagern für die rückkehrenden Flüchtlinge die Hauptaufgaben der Quäker. Wichtige Stationen entstanden zu diesem Zweck in Châlons, Bar-le-Duc, Semaizes, Troyes, Vitry-le-Francois und in Paris. Schließlich waren Quäker an der Somme vor der letzten großen Offensive des deutschen Heeres im Frühjahr 1918 an der Durchführung von Evakuierungsmaßnahmen und der Versorgung der Flüchtlinge beteiligt. Bei all diesen Tätigkeiten arbeiteten die Quäker eng mit der lokalen Bevölkerung, mit Bürgermeistern, Ärzten, Bauern und Handwerkern zusammen, deren Zahl die der etwa 100 Quäkerhelfer bei weitem überschritt. Die Erfahrungen dieser Zusammenarbeit dienten als Grundstock, auf der die folgenden Hilfsoperationen aufbauten. ${ }^{45}$

Frankreich war das bedeutendste, jedoch nicht das einzige Einsatzgebiet für den AFSC während des Ersten Weltkriegs. Vor allem Russland erschien zunächst als ein zweites mögliches Aktionsfeld, für das anfänglich der Aufbau einer größeren Hilfsmission mit amerikanischer Unterstützung erwogen wurde. Im Vergleich zu Frankreich waren die Bedingungen jedoch weniger günstig. „If we can imagine all the conditions in France intensified, with the added burden of appalling distances and seething political unrest, we have some picture of the need in Russia“, hieß es dazu in einem Lagebericht des AFSC. ${ }^{46}$ Anders als im Stellungskrieg im Westen war die Situation im Osten volatiler. Zu häufig wechselnden Frontverläufen und den davon ausgelösten Flüchtlingsbewegungen trat 1917 noch die wachsende politische Instabilität im Zarenreich, die Ende des Jahres in der Oktoberrevolution und der Machtübernahme der Bolschewiki gipfelte.

Die britischen Quäker, die seit 1916 im Land aktiv waren, hatten das AFSC hier zunächst um Unterstützung für ihre eigene 30-köpfige Mission gebeten. Nachdem man zunächst sechs weibliche Helfer im September 1917 entsandt hatte, entschied man in Philadelphia, dass die Lage insgesamt zu unsicher war. „The very facts which create the great need (in Russland, DM)“, hieß es im AFSC-Bulletin im Dezember 1917, „make it impossible or at least unsafe to send many workers to this field.“47 Daran änderte sich bis zum Ende des Krieges nichts Grundlegendes. Dennoch sollte die kleine angloamerikanische Mission in Buzuluk, in der Region von Samara im Süden Russlands, wenige Jahre später zum Ausgangspunkt für eine der größten Hilfsaktionen der Quäker werden. ${ }^{48}$

45 Six Months of Friends' War Relief Service, in: AFSC-Bulletin 8 (1917).

46 Six Months of Friends' War Relief Service, in: AFSC-Bulletin 8 (1917). Eine Zusammenfassung aller Aktivitäten in Frankreich auch bei Jones, Quakers in Action: Recent Humanitarian and Reform Activities of the American Quakers., 23-35.

47 Jones, Quakers in Action, 23-35.

48 Meeting of the Executive Board, in: AFSCA, AFSC Minutes 1918. 
Darüber hinaus waren einzelne AFSC-Helfer auch an anderen Kriegsschauplätzen wie Serbien und Griechenland an der Seite von britischen Einheiten im Einsatz. Gleichzeitig bedingte die Art des constructive service, für die das Komitee der Regierung ihre Kooperation anbot, auch die bewusste Entscheidung gegen andere humanitäre Anliegen. Für evangelikale Friends besonders schmerzhaft war etwa die Weigerung des AFSC, als Kanal für Quäkerhilfsgelder zugunsten der Opfer des Völkermordes an den Armeniern von 1915/1916 zu dienen. Das evangelikale Five Years Meeting, das über sein Mission Board enge Verbindung in die „bible lands“ (unter anderem die älteste Quäkermissionsschule in Ramallah, Palästina) unterhielt, reagierte deshalb mit einigem Unverständnis auf die Entscheidung des AFSC, dass diese Art von Hilfe als nicht vereinbar mit dem „distinctive work of Friends which this Committee is carrying on“ bezeichnete. Im Wesentlichen verstand sich das AFSC als ein Gremium, das Kriegsdienstverweigerern eine Gelegenheit für constructive service bieten wollte. Obwohl dies nicht unter allen Umständen (etwa in Russland) der Realität der Einsätze entsprach, ging es implizit darum, ein Bild aufrechtzuerhalten, in dem die Quäker als Teil des Kriegsaufwandes und im besten Fall des Frontgeschehens erschienen. ${ }^{49}$

Trotz seiner engen Bindung an das Amerikanische Rote Kreuz orientierten sich die AFSC-Helfer in Frankreich in vielfacher Hinsicht an den britischen Glaubensgenossen. Drei Jahre lang hatte man mit großem Interesse beobachtet, wie diese die Rolle der Quäker in einem total geführten Krieg zu bestimmen versuchten, in dem die Trennlinien zwischen Soldaten und Zivilisten zunehmend an Bedeutung verloren. Die oft schmerzhaften Erfahrungen, etwa der Kriegsdienstverweigerer oder beim Umgang mit einer oft feindseligen Öffentlichkeit, lieferten reichlich Anschauungsmaterial, aus denen die US-Quäker ihre Schlüsse zogen.

Auch das Interesse an einer Zusammenarbeit im Kriegsgebiet war nach dem amerikanischen Kriegseintritt auf beiden Seiten stark. Die Briten erhofften sich neben einer Verstärkung ihrer Einheiten nicht zuletzt einen finanziellen Schub für ihre Unternehmungen. Bereits unmittelbar nach Kriegseintritt der USA traten sowohl die FAU ${ }^{50}$ als auch das FWVRC ${ }^{51}$ mit der Bitte um Unterstützung an das

49 So hatte das Evangelistic and Church Extension Board des Friends Five Year Meeting of America einen Vertreter (Mead Kelsey) im American Committee for Armenian and Syrian Relief, dem Vorgänger des Near East Relief. Vincent Nicholson an Mead A. Kelsey 3/1/1918, in: AFSCA, General Administration (Armenian and Syrian Relief 1917-1918). Im breiteren Kontext siehe Watenpaugh, Bread from Stones: The Middle East and the Making of Modern Humanitarianism.

50 Die FAU bat im Juni um 50 Mann und finanzielle Unterstützung. Dies ließ sich jedoch nicht realisieren, da die FAU anders als das FWVRC direkt dem Militär unterstand. George Newman (London) berichtete, dass es kaum Aussichten für Amerikaner gab, in der FAU zu arbeiten, da 
AFSC heran. Aber auch das AFSC hatte ein unmittelbares Interesse an einer Kooperation mit den Briten. Eine solche Kooperation würde aus Sicht Philadelphias die beste Gewähr dafür bieten, dass die amerikanischen Quäker, ungeachtet ihrer formalen Unterordnung unter das Amerikanische Rote Kreuz, einen Teil ihrer religiösen Identität wahren und sichtbar machen konnten. Von den Briten, so die Überzeugung vieler im Young Friends Movement geschulter Quäker in den USA, konnte man zudem nicht nur in praktischer Hinsicht lernen. Vielmehr galten die britischen Quäker als Vorbild, da sie in Kriegszeiten neue Einheit und neuen Zusammenhalt gewonnen hätten. „A new birth of Quakerism“ sei in Großbritannien zu beobachten, hieß es in einem Artikel des AFSC-Bulletin im Herbst 1917.52 Das Projekt, das in Reichweite schien, war die organisatorische und spirituelle Erneuerung des Quäkertums aus dem Geist humanitären Dienstes in Kriegszeiten. Die Zusammenarbeit mit den Briten, so die Hoffnung im AFSC, würde auch die gespaltenen amerikanischen Quäker diesem Ideal näherbringen. ${ }^{53}$

Als Vincent Nicholson im August in einem Schreiben an die britischen Quäker in Frankreich die ersten amerikanischen Helfer ankündigte, sprach er ganz in diesem Sinn davon, dass die Bedeutung der gemeinsamen Arbeit in Zeit und Raum weit über den Einsatz in Frankreich hinausreiche. Grössere Einheit „,and more efficient fellowship“, in den USA und über den Ozean hinweg, würden die unmittelbare Folge des Einsatzes sein. ${ }^{54}$

Das Verhältnis der neu angekommenen amerikanischen Helfer zu ihren britischen Kameraden wurde in diesem Sinn anfänglich als „apprenticeship“ verstanden, aus der auf mittlere Sicht eine unabhängige American Friends Unit entstehen würde. ${ }^{55}$ Die hohe Wertschätzung der britischen Leistung drückte sich

sowohl die britische als auch die amerikanische Regierung einem solchen Plan ablehnend gegenüberstanden: Percy Alden (FAU) an Vincent Nicholson 4/6/1917, in: AFSCA, AFSC Minutes 1917 (General Meeting).

51 Es gab laut den Briten einen großen Bedarf an „doctors, nurses, workers on the land, motor mechanics, carpenters, builders or other relief workers“. FWVRC an Vincent Nicholson 25/6/1917, in: AFSCA, General Administration 1917 (American Red Cross, letters from Paris).

52 A new birth of Quakerism in Britain, in: AFSC-Bulletin 6 (1917).

53 A new birth of Quakerism in Britain, in: AFSC-Bulletin 6 (1917).

54 Vincent Nicholson an FWVRC (France) 24/8/1917, in: AFSCA, AFSC Minutes 1917 (General Meeting).

55 Eine direkte Zusammenarbeit mit den britischen Quäkern in einer gemeinsamen unabhängigen Quäkereinheit wurde kurze Zeit erwogen, dann jedoch rasch verworfen, auch weil die britischen Quäker ihrerseits von keiner größeren Organisation getragen wurden und ohnedies bereits an der Grenze ihrer Leistungsfähigkeit waren. Friends Construction and Relief Work in 
auch sichtbar in der Übernahme des englischen, achtstrahligen roten und schwarzen Sterns als gemeinsames Symbol der gemischten Quaker Units aus, bevor dieses zum Wahrzeichen des AFSC selbst wurde. ${ }^{56}$

Die Zusammenarbeit vor Ort gestaltete sich indes weniger einfach, als viele gehofft hatten. Während die Kooperation von Briten und Amerikanern auf der Führungsebene im Wesentlichen reibungslos verlief, war das Verhältnis innerhalb der neu gebildeten angloamerikanischen Einheiten von Anfang an von Konflikten geprägt. Die Gründe waren vielfältig: unterschiedliche „national and theological temperaments“, ${ }^{57}$ aber auch die Tatsache, dass sich die Zusammensetzung der Gruppe der amerikanischen Neuankömmlinge in wesentlichen Dingen von der der Briten unterschied. Die Helfer, die das AFSC nach Frankreich entsandte, waren in der Regel jünger und nach Nicholsons Einschätzung „perhaps not with the same depth of consecration to the task“ aufgrund der fehlenden Erfahrung mit der Arbeit unter Kriegsbedingungen. Diese Defizite konnten durch „boundless enthusiasm“ nur bedingt wettgemacht werden. ${ }^{58}$ Vielmehr war Letzterer oft insofern Teil des Problems, als die durch drei Jahre Einsatz hinter der Frontlinie abgehärteten Briten dem voluntaristischen Eifer der Neuankömmlinge oft mit Unverständnis und zuweilen Spott begegneten. ${ }^{59}$

Europe, in: AFSC-Bulletin 3 (1917); Concluding Report of our Mission send to France, in: AFSCBulletin 6 (1917).

56 Aus praktischen Gründen übernahmen die amerikanischen Neuankömmlinge zunächst auf der Kleidung das Zeichen des Roten Kreuzes, verwendeten aber darüber hinaus in ihrer Korrespondenz oder bei öffentlichen Gelegenheiten den achtstrahligen schwarz-roten „Quäkerstern“, das Erkennungszeichen britischer Quäker seit dem preußisch-französischen Krieg von 1870/71. Vincent Nicholson an Charles Norton (ARC) 26/7/1917, in: AFSCA, General Administration 1917 (American Red Cross, letters from Paris).

57 Greenwood, Friends and Relief, 206.

58 Vincent Nicholson an FWVRC (France) 24/8/1917, in: AFSCA, AFSC Minutes 1917 (General Meeting).

$59 \mathrm{Zu}$ den britisch-amerikanischen Gegensätzen siehe Frost, „Our Deeds Carry Our Message: The Early History of the American Friends Service Committee."Die beste zeitgenössische Quelle, welche die Gegensätze innerhalb der amerikanisch-britischen Zusammenarbeit in Frankreich aus Sicht des AFSC zusammenfasst, stammt dagegen aus dem Zweiten Weltkrieg. In einer breiten Strategiediskussion, in der es 1943 um die Ausrichtung der Quäkerhilfe nach dem Ende des Krieges ging beschrieb ein Teilnehmer, der selbst in Frankreich tätig gewesen war, die Zusammenarbeit als „an excessively difficult experiment.“ Einige Amerikaner hätten zu Anfang ihres Einsatzes die Einstellung gezeigt, als ob ,there hadn't been a war on, whereas the British had been through a form of martyrdom- jail, etc. and perhaps still felt that they should be martyrs. "Es habe anfänglich a „great many complications“ gegeben, „neither one understood the other, and they got into difficult tangles which were eventually straightened out and came out all right." In: Confidential Memorandum on post-war planning, Foreign Service Section 4 und 5/6/1943, in: AFSCA, AFSC Minutes 1943. 
Ein weiterer Faktor, der für Unstimmigkeiten sorgte, war, dass die Führungspositionen auf amerikanischer Seite sämtlich von Männern besetzt waren, bei den Briten dagegen auch Frauen an der Spitze einzelner Einheiten standen. Insbesondere zwischen Hilda Clark und Edith Pye einerseits, die auf britischer Seite die Hospitäler in Samoën und Châlons-sur-Marne leiteten, und James Babbitt andererseits kam es wiederholt zu Kompetenzrangeleien. Amerikanische Krankenschwestern weigerten sich zudem, unter der Führung von Pye zu arbeiten, der sie veraltete Methoden und mangelnde Professionalität vorwarfen. In der Tat wurde auf amerikanischer Seite von Anfang an Kritik an der angeblich fehlenden Effizienz und chaotischen Arbeitsweise der Briten laut, was die Gräben vertiefte. Was über diese Zwistigkeiten hinaus immer wieder deutlich wurde, waren unterschiedliche Selbstverständnisse. Auch als Folge seiner Ausrichtung am Vorbild des Amerikanischen Roten Kreuzes entwickelte das AFSC mit fortschreitender Dauer seines Einsatzes einen amerikanischen „humanitären Stil“, der einer vollständigen Verschmelzung von britischen und amerikanischen Quäkern in der oft beschworenen gemeinsamen „fellowship of service“ entgegenwirkte. Weitere Unterschiede traten hinzu: Politisch waren die Amerikaner, in ihrer Mehrzahl aus dem ländlichen Mittleren Westen stammend, in der Regel konservativer als ihre britischen Glaubensgenossen, unter denen viele Sozialisten waren. ${ }^{60}$

Was viele der Familien in den USA alarmierte, war zudem, dass die Briten, wie aus den Briefen der amerikanischen Helfer hervorging, eine weitaus liberalere Haltung an den Tag legten, was den Konsum von Alkohol und Tabak betraf. Das AFSC musste häufiger besorgte und empörte Schreiben der Eltern beantworten, die in der Regel Anhänger der Prohibitionsbewegung und selbst Totalabstinenzler waren. Diese fürchteten, dass ihre Söhne in Frankreich von ihren britischen Genossen moralischen Gefahren ausgesetzt seien (in aller Regel waren es Männer), gegen die sie das AFSC nicht genügend wappne. „The great mixing process“, in dem Quäker aller Art in einem gemeinsamen Werk zusammengeworfen seien, so Henry Scattergood in seiner Antwort an eine besorgte Mutter aus Ohio, verlange

60 Greenwood, Friends and Relief, 206. Ein weiterer Unterschied bestand offensichtlich in unterschiedlichen Erwartungen der britischen und amerikanischen Mitglieder der Hilfseinheiten an den Lebensstandard. Das oben bereits zitierte Memorandum aus dem Zweiten Weltkrieg berichtete davon: „Those who worked with American Friends in the relief period last time there was a feeling that the general American tendency for some of the workers to feel that they wanted a higher standard of office and living accommodation than the British were prepared to work with. Now equally, (...) some Americans felt that the British were prepared to work along under any circumstances and that wasn't efficient.“ In: Confidential Memorandum on post-war planning, Foreign Service Section 4 und 5/6/1943, in: AFSCA, AFSC Minutes 1943. 
allen Beteiligten Toleranz ab. „A great vision of living out a life of love“ sei diesen Preis wert. ${ }^{61}$ Darüber hinaus, so Scattergood, gelte Wein in Frankreich kaum als Alkohol und es sei in diesem Sinne auch ein Akt der Höflichkeit gegenüber der gastgebenden Kultur, die Vertretung eines prohibitionistischen Standpunktes nicht zu weit zu treiben. „I trust“, schloss er, „with these facts in mind the Friends will not allow these objections to rule their minds against the reconstruction work in general, because the good as a whole is vastly greater than these matters that some of us do not like but which we are helpless to control."62

Was dem AFSC im Kontrast dazu viel mehr Sorge bereiten musste, war, dass die eigenen Helfer durchaus andere Haltungen gegenüber Fragen von Krieg und Frieden an den Tag legten als ihre britischen Partner. Während die überwiegende Mehrzahl der Briten nämlich überzeugte und durch den Krieg in ihren Überzeugungen gestärkte Pazifisten waren, aus religiösen wie politischen Gründen, war dies bei einer großen Anzahl von Amerikanern schlicht nicht der Fall. Es machte sich bemerkbar, dass das AFSC als recht lose Klammer einer hinsichtlich ihrer sozialen Herkunft sowie ihrer politischen und religiösen Überzeugungen sehr heterogenen Gruppe fungierte.

In einem offenen Brief an das AFSC beschrieb Lewis Gannett, ein Mitglied des ersten Haverford-Lehrgangs, der als PR-Spezialist sowohl für das AFSC als auch für das Amerikanische Rote Kreuz tätig war, den Moment der Ankunft der ersten amerikanischen relief worker als einen Moment des ,intellectual and spiritual decline“. Innerhalb der amerikanischen Gruppe, so Gannett, seien Abenteurergeist und Drückebergertum wesentliche Antriebe, überzeugte Pazifisten finde man dagegen kaum. Viele seien von ihren religiösen Eltern dazu gedrängt worden, sich zu humanitärer Arbeit beim AFSC zu melden, wollten ansonsten aber am liebsten für Soldaten gehalten werden. Eine Reihe weiterer Briefe der amerikanischen Führungskräfte in Frankreich wie Charles Evans, Joseph Haines und Charles Rhoads bestätigten Gannetts Eindruck. Generell, so das Urteil, befänden sich unter den amerikanischen Neuankömmlingen viele unreife und wenig ge-

61 „I am sure even the most severe critics of these differences in habits would be immensely pleased by the underlying goodness and vision that most the English Friends really have." J. Henry Scattergood an Elise McCoy 3/7/1918, in: AFSCA, AFSC General Administration, (Criticism 1918).

62 „They (die Franzosen) do not understand why people object to drinking these light wines and teetotalers, in the sense that most of us are, are almost unknown among them, hence you can see that it has been quite natural that those who do not hold the strictest prohibition views should fall in with the ways of the country especially when the drinking water is bad." J. Henry Scattergood an Elise McCoy 3/7/1918, in: AFSCA, General Administration 1918 (Criticism 1918). 
festigte Persönlichkeiten. Der Eindruck sei verheerend und drohte nach Überzeugung vieler, die Position der Quäker insgesamt zu schädigen. ${ }^{63}$

Die Einheitsrhetorik des AFSC kaschierte nur mühsam das breite Spektrum der Motive, die junge Quäker zum Eintritt in die humanitären Hilfseinheiten bewogen hatten. Bereits seit Anfang des Jahres hatte das AFSC aus diesem Grund verstärkt an das Gewissen und den Quäkergeist der Helfer appelliert. In einem Schreiben vom Januar 1918 vermerkte Rufus Jones, die Wiederaufbauarbeit in Frankreich müsse im gleichen Maße als „manly effort“ und „spiritual service“ geleistet werden: „You cannot do your full service to France“, mahnte er, „unless you can help restore and refresh the spirit of those who have unspeakably suffered, and exhibit in your lives and in your words and in your work an underlying faith in eternal realities."64 Jones vermischte hier bewusst religiöse und militärische Metaphern, sprach vom „Heroismus“ der Tätigkeit, dem patriotischen Gehalt der humanitären Arbeit und betonte gleichzeitig das Quäkerspezifische der Aufgabe.

Kurz darauf wandte sich Jones mittels einer Broschüre unter dem Titel „The kind of men who should go to France“ auch an die Quäker zu Hause. ${ }^{65}$ Es sei wahr: In Frankreich brauche es „persons who can do things (kursiv im Original, DM)“. Doch sei dies nicht genug. Der Dienst jedes einzelnen müsse Ausdruck authentischen „Quaker faith and Quaker ideals“ sein.

An der Seite der Briten, denen der Weg der Gewissensverweigerung nicht in gleichem Maß offenstand, müssten die Amerikaner sich das Privileg des Dienstes durch Hingabe und Opferbereitschaft verdienen. Jeder, der von amerikanischer Seite dieser Gruppe ,with a lower motive and with a less noble purpose“ beitrete, gefährde nicht nur die Arbeit selbst, sondern, „what is far more serious, the tone and morale of the whole mission are affected“. Es sei wahr, räumte Jones ein, dass die amerikanischen Quäkerhelfer im Durchschnitt jünger und unerfahrener seien und entsprechend im Ganzen nicht die ,maturity of thought and insight and vision which marked the original band of workers“ erreichten. Gleichwohl müsse eines klar sein, schloss Jones mit einem flammenden Appell: „This is no affair for persons who are looking for some line of least resistance and are eager to escape something else which they dread. We want men of the best moral fibre and the soundest faith. It is not enough that the applicant can run a tractor or build a house or manage a sawmill. You must, first of all have a real man's soul inside.“

63 Gannett zitiert nach Frost, „Our Deeds Carry Our Message: The Early History of the American Friends Service Committee“, 24.

64 Rufus Jones an American Friends Unit 22/1/1918, in: AFSCA, AFSC Minutes 1918.

65 Broschüre: Rufus Jones, The Kind of Men who should go to France, in: AFSCA, General Administration 1918. 
Die Wirkung solcher Appelle blieb begrenzt. Ein Bericht von August 1918 mahnte nochmals in unmissverständlichen Worten einen „higher standard of qualification with particular emphasis upon the need for all men to be conscientious objectors“ an. Andernfalls drohe ein ,serious loss of our present position“ sowohl in der Gesellschaft selbst als auch mit Blick auf die Society of Friends. ${ }^{66}$ Über die tieferen Ursachen des Problems gab es wenig Illusionen: Am Ende reflektiere das Verhalten der amerikanischen relief worker den allgemeinen Zustand der Society of Friends in den USA. Nicht zuletzt bildeten sich hier die durchaus ambivalenten Haltungen zum peace testimony innerhalb des amerikanischen Quäkertums ab: „No one should expect the young to be better than the old“, hieß es dazu nüchtern. ${ }^{67}$ Zur selben Zeit stellte man fest, dass bei der bisherigen Arbeit der spirituelle Faktor bei der Auswahl und der Ausbildung der relief worker eine zu geringe Rolle gespielt hatte. In der Folge achtete man darauf, dem religiös-moralischen und dem professionellen Aspekt gleiches Gewicht zu geben. Auf der einen Seite sei es kein Selbstzweck, ,to save our boys who might otherwise be drafted“. Vielmehr müsse es darum gehen, „to send such men as will really be efficient workers in France“. Auf der anderen Seite wollte man den im Kern religiösen Charakter der Arbeit betonen und die Funktion der relief workers als „,silent witnesses against war" hervorheben. ${ }^{68}$

Doch auch Letzteres war nicht ohne Gefahren. Dass das AFSC im Laufe der Zeit erfolgreicher bei der Auswahl „echter“ Gewissensverweigerer für den Einsatz in Frankreich wurde, zeigte sich an den gegen Ende des Krieges gehäuften Beschwerden des Militärs, einzelne Quäkerhelfer ergingen sich in unerwünschten Aktivitäten. Ein Schreiben des Kriegsministeriums avisierte das AFSC in diesem Sinne, eine Reihe von Mitgliedern des amerikanischen Quäkerkontingents habe Anstoß erweckt (wörtlich hieß es, diese Personen hätten sich jeweils zur ,persona non grata“ gemacht), „owing to their freedom in expressing their convictions against all warfare. The French term is ,Defaitisme“ (defeatism) (...).“ Verbunden war der Hinweis mit der Mahnung, ,that any expression of pacifism of any kind cannot fail at this time to be exceedingly distasteful to those who have given their all to the cause of the Allies, as it is also contrary to the best interests of the American forces at the front." Das AFSC möge seine Kräfte im Feld in der gebotenen Schärfe anweisen, künftig alle Meinungsäußerungen zu unterlassen, die „however sincerely held are detrimental to the morale of the French people and

66 Report of Executive Secretary 22/8/1918, in: AFSCA, General Administration (Criticisms, 1918). 67 Frost, „Our Deeds Carry Our Message: The Early History of the American Friends Service Committee“, 26.

68 Frost, „Our Deeds Carry Our Message: The Early History of the American Friends Service Committee“, 26. 
(...) to the cause of America."69 Es war und blieb ein schmaler Grat, auf dem das AFSC mit seinem constructive service an der Front wandelte.

\section{Silent Representatives. Das AFSC und die humanitäre Mobilisierung in den USA}

Parallel zum Einsatz in den europäischen Kriegsgebieten erfüllte das AFSC zu Hause in den USA während des Ersten Weltkriegs eine weitere wichtige Funktion. Nolens volens erstreckte sich der constructive service, dem sich das Komitee verschrieben hatte, auch auf die Kriegsgesellschaft im eigenen Land. Das AFSC wurde Teil einer breiten Bewegung, durch die humanitäre Hilfe für die kämpfenden Truppen im Feld ebenso wie für Zivilisten in den mit den USA verbündeten Ländern zu einer Brücke wurde, die die heimische Bevölkerung mit dem Geschehen auf dem Kriegsschauplatz verband. Dabei war die Tätigkeit der Quäker an der „Heimatfront“ von Ambivalenz geprägt. Während in der Arbeit für die Kriegsdienstverweigerer die pazifistische Tradition und ein gewisses Maß an widerständigem Potenzial weiterlebte, erfüllte das AFSC auf anderer Ebene eine wichtige Funktion bei der Integration und Mobilisierung der Society of Friends für den Kriegsaufwand. ${ }^{70}$

Dabei verschaffte die Arbeit zugunsten von Kriegsdienstverweigerern den Quäkern zunächst eine Sonderrolle. Sie verfolgte einen grundsätzlichen Ansatz, der neben Angehörigen der Society of Friends sowohl Nicht-Quäker als auch Totalverweigerer einschloss. Dies entsprach der Überzeugung, dass die Gewissensentscheidung für Quäker als ein religiöses Gebot, darüber hinaus jedoch gerade nicht als „bequemes“ Privileg qua Mitgliedschaft in einer Quäkergemeinde gelten sollte. „We have to prove ourselves worthy of our exemption“, hieß es dazu in einer Erklärung. Das AFSC wollte die Verweigerung des Dienstes an der Waffe als individuellen Akt moralischer und spiritueller Standfestigkeit verstanden wissen, der ohne Ansehen der Motive zu respektieren war. In einer durch das AFSC gemeinsam mit anderen Friedensgruppen, wie der Fellowship of Reconciliation, dem

69 M. Churchill (War Department, Office of the Chief of Staff) an Vincent Nicholson 24/7/1918, in: AFSCA, General Administration 1918 (War Department).

70 Little, „An Explosion of New Endeavours: Global Humanitarian Responses to Industrialized War Fare in the First World War Era“; Irwin, „The Disaster of War: American Understandings of Catastrophe, Conflict and Relief“; Daniel Maul, „American Quakers, the Emergence of International Johannes Paulmann (Hg.) Humanitarianism and the Foundation of the American Friends Service Committee, 1890-1920,“ in Dilemmas of Humanitarian Aid in the Twentieth Century, (Oxford: Oxford University Press, 2016). 
Civil Liberties Bureau und der League for Democratic Control, veröffentlichten Stellungnahme forderte das Komitee im Januar 1918 die Gleichbehandlung aller Gewissensverweigerer, die als Männer definiert waren, ,who take the stand, that it is morally wrong for them to participate in this war" " ${ }^{71}$

Vor allem von dem Plan, auch Nicht-Quäker in die reconstruction units aufzunehmen, musste das AFSC jedoch früh Abstand nehmen, nachdem die Behörden bereits im Juni 1917 klargestellt hatten, dass nichtreligiöse Verweigerer grundsätzlich keine Anerkennung finden würden. Die überwiegende Mehrheit der Mitglieder der reconstruction units waren denn auch Quäker sowie Angehörige der übrigen „Friedenskirchen“, der Mennoniten und Brethren. Während sich die Arbeit für Nicht-Quäker und Totalverweigerer daraufhin auf die rechtliche Beratung und allgemeine Lobbytätigkeit zugunsten von Gewissensverweigerern beschränkte, beschäftigte sich ein eigens eingerichtetes Draft Committee mit jungen Quäkern, die von der Einziehung betroffen oder bedroht waren. ${ }^{72}$

Bei all dem agierte das Komitee in einer rechtlichen Grauzone. Zunächst ging es darum, Rechtssicherheit für jene wehrfähigen jungen Männer zu schaffen, die sich freiwillig, das heißt, ohne einen Einberufungsbescheid für das Militär erhalten zu haben, für den Dienst mit dem AFSC in Europa entschieden hatten. Im Juni 1917 bekam das AFSC bei einem Treffen mit Vertretern des War Department den Bescheid, dass Reisepässe an alle Quäkerhelfer unter der Bedingung ausgestellt würden, dass diese sich verpflichteten, im Falle der Einberufung unverzüglich ins Land zurückzukehren. Weiterhin offen blieb damit zunächst, ob die Betroffenen in einem solchen Fall auf einen positiven Bescheid und die Erlaubnis

71 Die Erklärung sah drei mögliche Formen des Dienstes vor: „a, noncombatant position with fighting units, b, noncombatant positions in hospitals here or abroad, c, alternative service ,either under control of the official representatives of an organized religious sect (...) or to work in government service not directly connected with carrying on the war'“. Für Totalverweigerer forderte das Papier die Internierung in speziellen Lagern statt in normalen Gefängnissen, wobei die Strafe nicht die Dauer des Krieges überschreiten sollte. Als Nachweis für eine Gewissensverweigerung sollte die Mitgliedschaft in Anti-Kriegsgruppen vor dem Kriegseintritt ausreichend sein. Die Untersuchung durch die Einziehungsbüros sollte individuell und ohne „coercive measures to determine conscience“ geschehen. Statement of Peace Groups including AFSC 9/1/1918; in: AFSCA, General Administration 1918 (Civil Liberties Bureau).

72 „We have endeavored in all ways“, hieß es in einer Zwischenbilanz Anfang des Jahres 1918, „to strengthen our young men in their conscientious convictions that all participation in the military machine is wrong and to help them stand firm for what they believe to be right." Für den Hintergrund Lester M. Jones, Quakers in Action: Recent Humanitarian and Reform Activities of the American Quakers (New York: Macmillan, 1929), 22. 
rechnen konnten, in den reconstruction units $\mathrm{zu}$ verbleiben. ${ }^{73}$ Erst im Oktober wurde diese Arbeit vom Kriegsministerium als „service necessary to the maintainance of national interest during the emergency“ anerkannt. Damit waren die Helfer des AFSC in Frankreich „on industrial grounds“ de facto vom Militärdienst befreit, und auf einer Stufe mit Personen, die kriegswichtige Tätigkeiten in der Heimat (etwa in der Rüstungsindustrie) vor der Einberufung schützten. ${ }^{74}$

Weitaus schwieriger war die Lage in jeder Hinsicht für diejenigen, welche die Freistellung vom Kriegsdienst beantragten, nachdem sie den Einberufungsbescheid erhalten hatten. Eine Entscheidung über den Ersatzdienst wurde über ein gutes Dreivierteljahr (bis in den März 1918) immer wieder vertagt, wohl vor allem, weil die Regierung dem Thema keine Öffentlichkeit verschaffen wollte. Das eröffnete denjenigen, die auf lokaler Ebene über entsprechende Anträge entschieden, einen breiten Spielraum. In der Regel hatte das Militär selbst das letzte Wort. Entscheidend war, dass der Antrag auf Verweigerung gestellt werden musste, nachdem der jeweilige junge Mann bereits in ein militärisches Ausbildungslager eingezogen worden war. Schikanen und Demütigungen prägten das Klima, dem die Eingezogenen in den Ausbildungslagern des Militärs ausgesetzt waren. Unverständnis von Seiten der Offiziere gegenüber den Gewissensverweigerern, Versuche, „to wear them down and crush their spirit“, und offene Feindseligkeiten von Seiten der anderen Rekruten bestimmten den Alltag. ${ }^{75}$

Fälle wie die von Howard Michener und Roland Cook, die für ihre Verweigerung militärischen Dienstes im Ausbildungslager Fort Flagler im Bundesstaat Washington in Einzelarrest und schließlich vor einem Kriegsgericht landeten, waren keine Seltenheit und wiederholten sich im ganzen Land, wie aus der Korrespondenz zwischen dem AFSC und dem Kriegsministerium deutlich hervorgeht. ${ }^{76}$ Als prominentesten Fall traf es im Sommer AFSC-Generalsekretär Tim Nicholson. Nachdem Nicholson die angebotene Befreiung vom Militärdienst abgelehnt hatte, verbrachte er den Rest des Krieges in einem Armeelager im Bun-

73 Protokoll des Besuchs des War Department: Treffen von Hollingworth Wood mit General Crowder, the Provost Marshall General 25/6/1917, in: AFSCA, General Administration 1917 (War Department, US Government).

74 AFSC Board Meeting 3/10/1917, in: AFSCA, AFSC Minutes 1917.

75 Erschwert wurde die Arbeit für die Gewissenverweigerer dadurch, dass sich die Quäker verstärkt mit dem Vorwurf des „Drückebergertums“ auseinandersetzen mussten, da gerade zu Beginn des Krieges einige Neueintritte in die Society of Friends durch junge Männer offensichtlich durch den Wunsch der Befreiung vom Militärdienst motiviert waren, AFSC Drafting Committee 21/ 3/1918, in: AFSCA, General Administration 1918.

76 Stanley Yarnall (AFSC) an Frederick Keppel (Assistant Secretary of War) 4/3/1918, in: AFSCA, General Administration 1918 (War Department). 
desstaat New York. ${ }^{77}$ Das AFSC versuchte die Moral der Verweigerer durch Briefkontakte und Besuche zu stärken. ${ }^{78}$ Den Männern wurde geraten, nach der Einberufung in die Camps die Offiziere bei jeder Gelegenheit auf die Existenz der reconstruction units zu verweisen und den Wunsch, diesen Dienst zu leisten. In die gleiche Richtung zielten die häufigen Eingaben an die lokalen Kommandanten der Militärcamps, die Betroffenen für die Wiederaufbaueinheiten freizustellen. ${ }^{79}$

Parallel zu dieser oftmals frustrierenden und wenig populären Tätigkeit für die Kriegsdienstverweigerer setzte sich das AFSC ab 1917 noch in ganz anderer Weise mit den Folgen der US-amerikanischen Kriegsbeteiligung auseinander. Über seine Publikationen, die dem Einsatz der Quäkerhelfer in Europa breiten Raum gaben, fungierte das AFSC als ein Medium, das Quäker in den USA direkt mit dem Kriegsgeschehen in Verbindung brachte. ${ }^{80}$ Damit gliederte sich das AFSC auf verschiedenen Ebenen in eine Entwicklung ein, in der humanitäre Hilfsorganisationen in den USA eine bedeutende Funktion bei der Unterstützung staatlicher Bemühungen um die Mobilisierung der Gesellschaft für den Krieg übernahmen.

Auch in diesem Bereich war das Amerikanische Rote Kreuz Vorreiter: Mit seinen Tausenden von lokalen Untergliederungen, die bis in die letzten Winkel des Landes reichten, übte es einen enormen Einfluss aus. Bereits lange vor dem amerikanischen Kriegseintritt und in einer Zeit, in der Neutralität die offizielle Politik darstellte, hatte das Rote Kreuz Pionierarbeit geleistet und über seine Hilfsaktionen eine Vielzahl von Menschen in den USA auf unmittelbare Weise mit dem Kriegsgeschehen in Verbindung gesetzt. Gemeinsam mit anderen Hilfsorganisationen, allen voran Herbert Hoovers Commission for the Relief of Belgium (CRB) trug das ARC zu einer „cultural mobilization“ bei - wie neuere Forschungen zeigen, auch bereits vor 1917 im überwiegenden Maße auf Seiten der Entente. ${ }^{81}$ Wenngleich die Quäker dieser Mobilisierung zuvor kritisch gegenüberstanden, gliederten sie sich nach dem Kriegseintritt auf ihre eigene Weise ein. Das Rote Kreuz lieferte in vielfacher Hinsicht das Vorbild.

77 Seinen Posten übernahm der aus Indiana stammende und seit 1908 als Pastor einer Quäkergemeinde in Boston tätige Wilbur Thomas.

78 Im März erging ein entsprechender Aufruf an alle Quäker zu Besuchen in den lokalen Militärcamps, AFSC Drafting Committee 21/3/1918, in: AFSCA, AFSC Minutes 1918.

79 AFSC Drafting Committee 9/1/1918, in: AFSCA, AFSC Minutes 1918.

80 Daniel Maul, „Appell an Das Gewissen Der Völker - Fridtjof Nansen, Der Internationale Humanitarismus Und Die Russische Hungerhilfe 1921-23 „Themenportal Europäische Geschichte $46-60$.

81 Elisabeth Piller, „American War Relief, Cultural Mobilization, and the Myth of Impartial Humanitarianism“, in: Journal of the Gilded Age and Progressive Era 17, 4 (2018), 619-635. 
Eine der ersten Folgen war die Professionalisierung der humanitären Infrastruktur des AFSC, etwa in der Einrichtung eines permanenten Sekretariats, einer generellen Straffung der Organisationsstruktur sowie der Einrichtung einer Presse- und PR-Abteilung. An der Führungsspitze traten religiöse Autoritäten wie Rufus Jones nach den ersten Monaten zugunsten von Rechtsanwälten, Unternehmern und Sozialarbeitern in den Hintergrund..$^{82}$ Auch in der Ausbildung von Quäkerhelfern für den Dienst in Europa rückten professionelle Gesichtspunkte in den Vordergrund vor die ideellen. Die Quäker-Colleges im Land wurden nun zum Rekrutierungsfeld für Ärzte, Architekten, Logistikexperten und Ingenieure, welche die Führungspositionen innerhalb der relief units ausfüllten.

Bei der Auswahl der Kandidaten für den Dienst in Frankreich im Ausbildungscamp in Haverford wurde der Gewissensfaktor zunehmend nachrangig. Unter den ersten „Rekruten“ befanden sich dann auch nur wenige Gewissensverweigerer, und nur fünf Mitglieder der radikalpazifistischen Fellowship of Reconciliation. Von Seiten des AFSC war man nun umso mehr darauf bedacht, dass unter den Ausgewählten auch genügend Personen waren, die für die konkrete Tätigkeit in Frankreich relevante Fähigkeiten besaßen, etwa in der Landwirtschaft. Der religiöse Aspekt spielte anfangs eine gänzlich untergeordnete Rolle. Daneben wurde aber offensichtlich auch ein geografischer Schlüssel bei der Auswahl angewandt. In etwa gleich viele Teilnehmer kamen von der Ostküste und aus dem Mittleren Westen, daneben auch einige wenige aus Quäkergemeinden in Kalifornien und Oregon. Zweifellos spiegelte sich hierin der integrative Ansatz des AFSC wider, das trotz allem als Vertretung aller Quäker im Land fungieren wollte. ${ }^{83}$

Auch in anderen Bereichen war die Orientierung am Roten Kreuz augenscheinlich. Gerade wegen der pazifistischen Färbung des AFSC war auffällig, wie sehr es sich äußerlich dem militärischen Habitus des ARC anglich: Die Tatsache, dass Haverford die Auszubildenden nach dem Vorbild der quasi-soldatischen Uniformen der Rotkreuzhelfer mit einer stark an eine militärische Kluft erin-

82 Frost, „Our Deeds Carry Our Message: The Early History of the American Friends Service Committee", 12.

83 William Frost liefert eine Reihe von weiteren Hinweisen auf die Zusammensetzung der Gruppe. Die grösste Gruppe stammte demnach aus Pennsylvania, gefolgt von Indiana und Ohio, zwei weiteren Staaten mit hohem Anteil an Quäkern. Mehr als die Hälfte der Teilnehmer war zwischen 21 und 25 Jahre alt, ein weiteres Viertel war 20 oder jünger, lediglich drei Prozent über 30. Vom Bildungsgrad waren rund zwei Drittel der „Rekruten“ entweder College Studenten oder verfügten bereits über einen College-Abschluss. Zur selben Zeit waren landwirtschaftliche Erfahrung unter den Teilnehmern weit verbreitet. Frost, „Our Deeds Carry Our Message: The Early History of the American Friends Service Committee“, 14-18. 
nernden Einheitskleidung ausstaffierte, ließ sich in zwei unterschiedliche Richtungen interpretieren. Zum einen war die Uniformierung fraglos ein wichtiger und bewusst vollzogener Schritt, der den Kandidaten helfen sollte, eine corporate identity zu entwickeln. Andererseits war sie ein ebenso wichtiges Signal nach außen, indem sie den Anspruch der Quäker sichtbar unterstrich, einen Dienst zu leisten, der dem des Frontsoldaten oder des Rotkreuzhelfers an patriotischem Geist in nichts nachstand, und der humanitäre Arbeit als Teil des Kriegsaufwandes inszenierte.

Besonders auffällig wirkte sich die Angleichung an den vom Roten Kreuz bestimmten Trend mit Blick auf die Rolle von Frauen in der Quäkerhilfe aus. Nahmen Frauen wie gezeigt traditionell eine wichtige Rolle in der Quäkerhilfe ein, so nahm ihre Bedeutung nach Kriegsbeginn stetig ab. Auf diese Weise näherte sich das AFSC mehr und mehr an die Strategie des ARC an, die humanitäre Arbeit als eine einem Manne angemessene, ja per se maskuline Tätigkeit zu etablieren suchte. ${ }^{84}$ Zwar eröffnete das Rote Kreuz Frauen einen der wenigen Räume, sich beruflich zu verwirklichen, etwa als Ärztinnen, Krankenschwestern oder Sozialarbeiterinnen. In der Führungsspitze dagegen wurden alle wichtigen Positionen von Männern besetzt. Das AFSC glich sich dem nach Kriegsbeginn rasch an. Von den Untergliederungen des Komitees wurde im Sommer 1917 lediglich ein Teilbereich von einer Frau (Rebecca Carter) geleitet, bezeichnenderweise das Committee on Women's Work. Das Executive Board des AFSC als eigentliches Machtzentrum bestand zum gleichen Zeitpunkt nahezu ausschließlich aus Männern.

Parallel spielten amerikanische Quäkerinnen im Vergleich zu ihren britischen Glaubensgenossinnen auch im Feld eine untergeordnete Rolle. Dabei war das AFSC anfänglich durchaus darum bemüht gewesen, Frauen eine prominente Rolle innerhalb der geplanten Hilfseinsätze einzuräumen. ${ }^{85}$ Von diesen Plänen kam das AFSC jedoch ab, als klar wurde, dass dies die Zusammenarbeit mit dem ARC in Frage stellen würde, das eine deutliche Präferenz für männliche relief worker zeigte. ${ }^{86}$ Inwieweit dieser Druck zur Anpassung an die Politik und den

84 Zur ambivalenten Rolle der Frauen im ARC, das Möglichkeiten der Partizipation eröffnete und gleichzeitig Frauen in der Führung (trotz der herausragenden Rolle der Gründungsfiguren Clara Barton und Mabel T. Boardman) an den Rand drängte, Julia Irwin, Making the World Safe: The American Red Cross and a Nation's Humanitarian Awakening (New York: Oxford University Press, 2013).

85 Man ging zunächst von einem hohen Bedarf an weiblichen Helfern aus und begann mit Vorbereitungen, diese auch in die Kurse in Haverford aufzunehmen. Meeting 4/6/1917, in: AFSCA, AFSC Minutes 1917 (General Meeting).

86 Man entschied sich im Juni, zunächst mit der Aufnahme weiblicher Helfer für die Ausbildung in Haverford fortzufahren, allerdings ,,with such enrollment with very little public statement until 
humanitären Stil des Roten Kreuzes für diese Entwicklung den Ausschlag gab, ist im Einzelfall schwer zu bemessen. Aufs Ganze betrachtet ließ sich in der Arbeit des AFSC ein klarer Trend zur Trennung der Geschlechterrollen beobachten, wobei sowohl die Führung des Komitees als auch der Hilfseinsatz an der Front ein deutlich männliches Gesicht trug, während Quäkerinnen in der Regel an der „Heimatfront“ oder als Krankenschwestern unter der Ägide des Roten Kreuzes dienten. Wiederholt unternahm das AFSC in der Folgezeit Anläufe, weibliche relief worker in die Kriegsgebiete zu entsenden, letztlich jedoch ohne Ergebnis. ${ }^{87}$

Auch in anderen Bereichen lassen sich Parallelen feststellen: Durch seine Publikationen stellte das AFSC eine Brücke zum amerikanischen Kriegseinsatz sowie zu den Empfängern der Hilfe im Kriegsgebiet her und schloss die amerikanischen Quäker in den breiteren globalen Kontext amerikanischer Politik ein. In diesem Sinne fügte sich die Arbeit des AFSC gegenüber den 125.000 amerikanischen Quäkern ganz in die Strategie der US-Regierung und die an viele Millionen gerichteten Unternehmungen des Roten Kreuzes zur Mobilisierung der Kriegsgesellschaft ein. Wenn der Erfolg eines Landes in einem total geführten Krieg von der Fähigkeit abhing, „to make all citizens willing conscripts for war service“, ${ }^{88}$ dann war diese Arbeit zu Hause ebenso bedeutend wie die Versorgung von Soldaten und Zivilisten am Kriegsschauplatz.

Von Anbeginn mahnte das AFSC alle amerikanischen Quäker, eine Opferbereitschaft „comparable to the sacrifice of others“ $\mathrm{zu}$ zeigen. ${ }^{89}$ Gemeint war vor allem die finanzielle Unterstützung, wie eines der Kriegsbulletins mit der einleitenden Überschrift „the Universal Service for all Friends is that of financial contribution“ verdeutlichte. ${ }^{90}$ Wer konnte, sollte jedoch darüber hinaus einen aktiven Dienst leisten. Damit waren insbesondere die Frauen angesprochen: Es wurde für die Einrichtung von Näh- (sewing), Strick- (knitting) und Einweck-Vereinen (canning clubs) geworben. Unter der Überschrift „The obligation of women Friends for emergency service“ wurden alle Frauen dazu aufgerufen, ihren Anteil zu leisten,

the prospects of sending women were brighter“. General Meeting, 25/6/1917, in AFSCA, Minutes 1917.

87 Auch das Ansinnen einer Gruppe von jüdischen Krankenschwestern, die aus pazifistischer Überzeugung nicht mit dem Roten Kreuz arbeiten wollten und stattdessen dem AFSC in Russland ihre Dienste anboten, konnte aufgrund der als zu schlecht eingestuften Sicherheitslage nicht realisiert werden. Rebecca Carter, Bericht des Committee on Women’s Work 3/10/1917; 15/1/1918, in: AFSCA, General Administration 1918 (Executive Board 1918).

88 Proctor, Civilians in a World at War, 14.

89 Service in Financial Contribution, in: AFSC-Bulletin 2 (1917).

90 Service in Financial Contribution, in: AFSC-Bulletin 2 (1917). 
damit sowohl das Land wie auch die Society of Friends ihrer Mission gerecht werden könne. ${ }^{91}$

Die Publikationen des AFSC ließen keinen Zweifel, dass es sich bei dieser Arbeit um ein Werk von nationaler Bedeutung handelte. So hieß es im Dezember 1917, die sewing clubs der Society of Friends seien als Beleg für die Verbundenheit zwischen französischen und amerikanischen Frauen in schwierigen Zeiten zu sehen. ${ }^{92}$ Nirgends wurde deutlicher, dass das Nähen für europäische Zivilisten als Akt des praktizierten Patriotismus begriffen wurde, als in einer im selben Monat vom Committee on Women's Work herausgegebenen Anleitung zur Herstellung von Kleidungsstücken.

Letztere sollten, wie bereits die Überschrift erkennen ließ, nicht als bloße Liebesgaben, sondern als nichts weniger als „our representatives in France“ gesehen werden. So wie die Quäkerhelfer, die in Frankreich ihren Dienst taten, dort als Botschafter ihres Landes agierten, so sei auch die Kleidung, die Frauen im ganzen Land für die Notleidenden in Frankreich fertigten, eine „silent army of representatives“, die das Bild der USA im Ausland präge.

„Those garments of all sizes and kinds which are worn and used by thousands of refugees and observed by thousands more. What of their influence? Are they representing us as we would wish to be known (?)“, lautete die rhetorische Frage. Ebenso wie gut genähte, hochwertige Kleidung die Herzen höher schlagen lasse „with the thought of a great nation beyond the sea“, so sei schlecht und sorglos verarbeitete Ware dazu geeignet, ein ungünstiges Licht nicht nur auf die Näherinnen und die Quäker, sondern auf das Land als Ganzes zu werfen. Alle müssten sich darüber im Klaren sein, dass sie persönliche Verantwortung für das Bild der USA im Ausland trugen. „A test of a person, a society or a nation“, so hieß es weiter, „is the way they give to the needy.“ Die Frage, die an jedes Kleidungsstück zu stellen war, lautete in diesem Sinne nicht nur, ob dieses dem persönlichen oder quäkerspezifischen Standard entspreche, sondern auch und nicht zuletzt: „Is it worthy of America?“93

Humanitäre Hilfe wurde auf diese Weise zum Schaufenster stilisiert, durch das andere die Quäker und die USA gleichermaßen wahrnehmen würden. In jedem Fall entsprachen die Nähclubs in ihrer Tätigkeit der Zielsetzung, die dem ARC bei seiner Arbeit an der „Heimatfront“ vorschwebte. Quäkerfrauen strickten für

91 Service in Sewing and Knitting, in: AFSC-Bulletin 4 (1917). Zusammenfassend in What part can women friends have in Friends War Relief Service?, in: AFSC-Bulletin 9 (1917).

92 Six Months of Friends War Relief Service, in: AFSC- Bulletin 8 (1917).

93 „Our representatives in France“, in: AFSC-Bulletin 12a (1918). 
europäische Zivilisten, wie es ein früher Chronist des Einsatzes zusammenfasste, „as other women sewed and knitted for the boys in Khaki“. ${ }^{94}$

Die Annäherung an den humanitären Stil des ARC war für die Quäker nicht ohne Fallstricke: War die Anpassung an das ARC, dessen Staatsnähe und patriotische Ausrichtung außer Frage standen, auf der einen Seite die wirksamste Versicherung gegen den Vorwurf der Illoyalität, so war es aus anderem Blickwinkel mindestens ebenso wichtig, eine gewisse Distanz zu wahren. Aussagen führender Vertreter des ARC wie Ernest Bicknell - der die Quäker für ihren humanitären Dienst in Frankreich mit den Worten belobigte, kein zweiter habe der amerikanischen Armee mehr gedient, sei mithin „more useful and more helpful toward winning the war" - brachten die Gefahr mit sich, das AFSC in den Augen vieler Quäker zu nahe an die Militärmaschinerie zu rücken. ${ }^{95}$ Dies berührte zweifellos einen wunden Punkt. Während die Zwänge des Krieges viele der diesbezüglichen Bedenken in den Hintergrund drängten, blieb vielerorts ein Unbehagen erhalten, das bei Kriegsende unweigerlich aufs Tapet kommen würde. Die Überlegung war folglich einfach: Sollte die gemeinsame humanitäre Arbeit den Quäkern zu neuer Einheit verhelfen, musste die Arbeit auch jetzt bereits ein erkennbares Quäkerprofil tragen. Dieser identitätsstiftende Markenkern ließ sich freilich nur dann aufrechterhalten, wenn die Arbeit des AFSC auch zu Hause nicht vollständig in derjenigen des Roten Kreuzes aufging. Dies hatte sowohl einen ideellen als auch einen finanziellen Aspekt.

„Friends should concentrate their support back of our own work“, hieß es hierzu bereits in einem der ersten Bulletins des AFSC im Mai 1917. Es wurde darauf hingewiesen, dass es sich bei den mit dem Roten Kreuz getroffenen Arrangements, wonach offiziell alle durch das AFSC für die humanitäre Arbeit im Ausland eingeworbenen Gelder als Teil der Rotkreuzkampagne gelistet werden mussten, um ,a matter of form only“ handele. Es wäre fatal, so hieß es weiter, wenn daraus die Ansicht abgeleitet würde, es sei gleichgültig, ob die Spenden über das AFSC oder direkt an das Rote Kreuz kämen. In Wahrheit habe man sich verpflichtet, die Arbeit der Quäkerhelfer alleine aus den eigenen Mitteln zu finanzieren. Dies bedeute ,as a natural result the need for Friends to make their Red Cross contribution to our own work through our own channels“ (kursiv im Original, DM). Dies im Kopf zu behalten, sei „essential to the success of the work we have been asked to do“. Um sichtbar zu bleiben, wurde in diesem Sinne nochmals mit großem

94 Jones, Quakers in Action: Recent Humanitarian and Reform Activities of the American Quakers, 21.

95 Ernest Bicknell (ARC) an Vincent Nicholson 8/3/1918, in: AFSCA, General Administration 1918 (American Red Cross). 
Nachdruck betont, dass ,the whole of Friends contribution in support of our own work“ gefragt war: „The tasks we face cannot possibly be accomplished unless this is done." "96

Dass es dem AFSC nicht alleine um die Finanzmittel ging, wurde spätestens deutlich, als sich das Komitee wenig später auch an die Frauen mit der Bitte wandte, eigene Quäkernähclubs zu gründen „in preference to work under local Red Cross or other organizations“. ${ }^{97}$ Wie ein Memorandum des AFSC im Januar 1918 feststellte, wurde der Anspruch der Quäker, Spenden für ihre eigene Arbeit durch eigene Kanäle zu sammeln, zu einem steten Quell von „friction and misunderstanding“ mit den lokalen Rotkreuzvertretungen.

„In the past the statement of Friends that they were contributing to Friends work“, so hieß es hier, „had not satisfied local Red Cross people as a reason for not giving largely to the Red Cross (...). “98 Verkompliziert wurde die Lage durch den Umstand, dass es dem AFSC nicht gelang, die Arbeit seiner eigenen reconstruction units komplett aus eigenen Mitteln zu bestreiten, sondern dass man von Quäkerseite auf Zuschüsse des ARC angewiesen war. Umgekehrt war es gegenüber der eigenen Klientel schwer zu vermitteln, dass das AFSC die Kosten für sämtliche Quäkerhelfer in Frankreich zu tragen beanspruchte, weil nominell ein Unterschied bestand zwischen dem Teil der Helfer, der in der Mission Angloamericaine, und jenem, der unter dem Dach des ARC arbeitete. Das AFSC steckte bei seinen Spendenaufrufen vor diesem Hintergrund in einem grundsätzlichen Dilemma: Eine eigenständige USA-weite Spendenkampagne war kaum denkbar, wäre diese doch in Konkurrenz zum nationalen Aufruf des ARC getreten und hätte dessen Monopol in Frage gestellt. Die Idee wurde dann auch früh zu den Akten gelegt. Als Teil der Rotkreuzkampagne drohten die Quäker gleichsam unsichtbar zu bleiben. ${ }^{99}$

Die Suche nach einem Übereinkommen, das ein Maximum an Spenden für die spezifische Arbeit der Quäker und dabei möglichst wenig böses Blut im Verhältnis mit dem ARC generierte, bildete in diesem Sinn eine Konstante in der Arbeit des AFSC. Sowohl den Quäkergemeinden im Land als auch der Spitze des ARC in Washington gegenüber verteidigte man den Standpunkt, dass beide Seiten am meisten gewannen bei einem Arrangement, bei dem die Quäker ihre vollständige Unabhängigkeit bewahrten: „More money could be raised from Friends if it went

96 Service in Financial Contribution, in: AFSC-Bulletin 2 (1917).

97 Service in Sewing and Knitting, in: AFSC-Bulletin 4 (1917).

98 Memorandum „Our relationship with the Red Cross“18/1/1918, in: AFSCA, AFSC Minutes 1918 (Executive Board).

99 Minutes of the AFSC Board 18/1/1918, in: AFSCA, AFSC Minutes 1918 (Executive Board). 
through Friends channels“, lautete dabei die beharrlich verfolgte Argumentationslinie. ${ }^{100}$

Diese Position bekräftigte im Mai 1918 eine Sonderausgabe des AFSC-Bulletins zum Verhältnis von Quäkern und Rotem Kreuz unter der Überschrift „Important Points to remember“. Die Quäker, wiewohl weiterhin Teil der nationalen Rotkreuzspendenkampagne, behaupteten ihre komplette finanzielle Eigenständigkeit: Quäkergeld würde durch Quäkerkanäle allein für Quäkerhilfe zur Verfügung stehen. ${ }^{101}$

Kaum ein Bereich spiegelt die Gespaltenheit des AFSC in diesen Fragen besser als die Öffentlichkeitsarbeit. Durch innovative Werbekampagnen war es dem Roten Kreuz während des Krieges gelungen, eine breite Öffentlichkeit mit der Arbeit der Organisation vertraut zu machen. Plakataktionen, die Veranstaltung von fund-raisers sowie insbesondere die Herausgabe einer Mitgliederzeitschrift, hatten ihren Anteil bei der Entstehung einer Massenorganisation. Aufbauend auf diesen Erfahrungen begann auch das AFSC bereits im Mai 1917 damit, ein monatliches Bulletin herauszugeben, dass an alle Quäkergemeinden im Land versandt wurde. Durch das Bulletin schuf das Komitee ein Medium, über das Quäker in den USA regelmäßig über die Einsätze der Quäkereinheiten in Frankreich und Russland informiert wurden. Es erfüllte damit eine wichtige Funktion bei der Identitätsstiftung unter den amerikanischen Friends und reichte in seiner Bedeutung über den Krieg hinaus. ${ }^{102}$

Ein weiterer Teil der Öffentlichkeitsarbeit richtete sich an die Außenwelt, von der bestenfalls Unverständnis für den Standpunkt des AFSC zu erwarten war. Hier galt es, über die Aktivitäten der Quäker aufzuklären, nachdem erste Bemühungen in diesem Bereich als unzureichend erkannt worden waren: Eine „more extensive and thorough policy of publicity of Friends war relief work" mahnten einige Mitglieder des AFSC board in diesem Sinne im März 1918 an. Es sei an der Zeit, die sonst übliche Bescheidenheit aufzugeben. Vor allem sollte die grundsätzliche Position der Quäker offensiver vermittelt werden, und zwar „both in order to clear up misunderstanding and to keep burning the all too obscure light of the Friends position“. Dies sei nicht zuletzt notwendig, um Quäkern eine Hilfestellung zu leisten, „who are forced to undergo the persecution of public opinion and often the loss of position because of their principles"“. ${ }^{103}$ Wiederholt wurde die Öffentlichkeitsarbeit in der Folge personell aufgestockt und vor allem die Pressearbeit intensiviert, die vornehmlich darauf zielte, das Wissen um die Arbeit der Quäker

100 Minutes of the AFSC Board 18/1/1918, in: AFSCA, AFSC Minutes 1918 (Executive Board).

101 Friends and the Red Cross campaign, in: AFSC-Bulletin 14 (1918).

102 Die erste Ausgabe erschien im Mai 1917.

103 Meeting of the Executive Board 21/3/1918, in: AFSCA, AFSC Minutes 1918 (Executive Board). 
in Frankreich in Zeitungen und Magazinen quer durch die USA zu verbreiten. Zu diesem Zweck wurden viele persönliche Erfahrungsberichte gesammelt, in denen das Bild prinzipiengeleiteter, selbstloser Arbeit übermittelt werden sollte. ${ }^{104}$

\section{The real work has just begun. Das AFSC nach dem Waffenstillstand}

Mit dem Waffenstillstand im November 1918 endete die erste Etappe der Quäkerhilfe. Nach und nach verließen nun die meisten der Eingezogenen die Einheiten und kehrten nach Hause zurück. Dennoch stoppte die Hilfe nicht sofort. Unmittelbar vor Kriegsende hatten die französischen Behörden die Quäkermission um Hilfe beim Wiederaufbau von 44 Dörfern in der Umgebung der Schlachtfelder von Verdun gebeten. Darüber hinaus wurden auch die meisten Aktivitäten beim Bau von Notunterkünften oder in der Landwirtschaft über das Kriegsende hinaus zunächst mit kleinerer Belegschaft weitergeführt. Britische und amerikanische Quäker engagierten sich in Verdun und an anderen Orten bei der Repatriierung von Flüchtlingen, richteten Kooperativen ein, in deren Läden sich die Rückkehrer mit dem Nötigsten ausstatten konnten. Das Einkommen aus dieser Arbeit finanzierte einen Teil der übrigen Aktivitäten, wie etwa den Unterhalt der Geburtsklinik von Chalons, die noch bis 1920 unter Aufsicht der Quäker verblieb, ehe sie in französische Verwaltung überging.

Zur selben Zeit änderte sich der Status der Quäkerhilfe: Die Bindung an das ARC endete und die Verantwortung für die Versorgung der Zivilbevölkerung ging von amerikanischer Seite Anfang 1919 auf die neu gegründete American Relief Administration (ARA) über. Damit endete auch automatisch die formale Verbindung mit dem amerikanischen Militär. ${ }^{105}$

Für den AFSC war dies der Moment für eine Bestandsaufnahme, auch da die ursprüngliche Grundlage, auf der das Komitee gearbeitet hatte, mit dem Ende des Krieges wegfiel. Damit war es für die Führungsspitze nun wichtig, das fortgesetzte Engagement in Europa vor der eigenen Klientel, sprich: den Quäkergemeinden, die das Komitee trugen, zu rechtfertigen. In einem Memorandum von Ende November, knapp zwei Wochen nach dem Abschluss des Waffenstillstands äußerte das AFSC die Überzeugung, dass „,the real work of the AFSC has just begun“. Es sei

104 Im Mai 1918 kam das AFSC dennoch zu einer ambivalenten Bilanz. Besonders kritisch wurde gesehen, dass kleinere Einheiten wie jenes des Smith College weitaus mehr Publizität erreichten. Der AFSC-Stern als „brand“ müsse noch bekannter gemacht werden. AFSC Board 23/5/1918, in: AFSCA, AFSC Minutes 1918 (Executive Board).

105 Zur ARA siehe auch das folgende Kapitel zur Quäkerhilfe in Deutschland. 
dem Komittee zu zeigen gelungen, dass es möglich war, patriotischen Dienst mit den spezifischen Werten und Überzeugungen der Society of Friends in Einklang zu bringen.

Durch das Ende des Krieges eröffneten sich nun neue Möglichkeiten: Befreit von militärischen Zwängen und dem Druck einer feindseligen Öffentlichkeit gab es nun Gelegenheit, ,to show the world that we and our young people are not trying to avoid work of world importance“. Erst die Weiterführung der Arbeit werde den letzten Beweis erbringen, dass die Quäkerhelfer auf Basis von klaren Überzeugungen und patriotischem Verantwortungsgefühl handelten. Die Hilfe beim Wiederaufbau sei in dieser Hinsicht ein starkes Signal.

Das Kriegsende bot darüber hinaus auch einen Anlass, den spirituellen Aspekt der Quäkerhilfe wieder stärker in den Mittelpunkt zu rücken. Nun war der Moment, den Nachweis zu erbringen, dass „seeds and tools are only the outward expression of our real message“. Wenngleich missionarische Arbeit wie überhaupt jede religiöse Arbeit im engeren Sinn bis zu einem endgültigen Friedensschluss weiterhin untersagt blieb, eröffneten sich neue Spielräume alleine durch den gewandelten Charakter der Arbeit. Das „Heilen der Kriegswunden“ war eine Möglichkeit, den religiösen Kern der Quäkerhilfe freizulegen: „to fulfill religious commitment by humanitarian endeavours“. ${ }^{106}$

Schließlich fehlte auch der Appell an den neu gewonnenen Zusammenhalt nicht, den das AFSC mit seiner Arbeit verkörpere. „For the first time in its history“, erklärte das November-Memorandum, „the Society of Friends are united in one work(...).“ „It is a strength to all to know“, so weiter, „that there is one organization that can give concrete expression to that part of their faith which they hold in common. “107

Diese Einheit, repräsentiert durch das AFSC, war ein Gut, ein zartes Pflänzchen, geboren aus der Not des Krieges, das es nun unter geänderten Bedingungen zu bewahren und zu pflegen galt. Eine neue Identität aus dem Geist humanitären Handelns für die amerikanischen Quäker zu erreichen, größere Einheit und spirituelle Erneuerung, aber auch einen quäkerspezifischen Beitrag zur neuen globalen Rolle der USA als Weltmacht zu leisten - das war das Projekt, dem sich das AFSC verschrieben hatte. Schon sehr bald würde sich in Deutschland die Gelegenheit ergeben, die Tragfähigkeit dieses Anspruchs auf die Probe zu stellen.

106 Minute on the Future Work of the AFSC 27/11/1918, in AFSCA, AFSC Minutes 1918 (General Meeting).

107 Minute on the Future Work of the AFSC, 27/11/1918, in AFSCA, AFSC Minutes 1918 (General Meeting). 